\title{
Estimating age-time-dependent malaria force of infection accounting for unobserved heterogeneity
}

\author{
L. MUGENYI ${ }^{1,2}$, S. ABRAMS ${ }^{2}$ AND N. HENS ${ }^{2,3}$ \\ ${ }^{1}$ Infectious Diseases Research Collaboration, Plot 2C Nakasero Hill Road, Kampala, Uganda \\ ${ }^{2}$ Center for Statistics, Interuniversity Institute for Biostatistics and statistical Bioinformatics, UHasselt (Hasselt \\ University), Diepenbeek, Belgium \\ ${ }^{3}$ Centre for Health Economics Research and Modelling Infectious Diseases, Vaccine and Infectious Disease \\ Institute, University of Antwerp, Antwerp, Belgium
}

Received 29 December 2016; Final revision 23 March 2017; Accepted 2 June 2017;

first published online 5 July 2017

\section{SUMMARY}

Despite well-recognized heterogeneity in malaria transmission, key parameters such as the force of infection (FOI) are generally estimated ignoring the intrinsic variability in individual infection risks. Given the potential impact of heterogeneity on the estimation of the FOI, we estimate this quantity accounting for both observed and unobserved heterogeneity. We used cohort data of children aged $0 \cdot 5-10$ years evaluated for the presence of malaria parasites at three sites in Uganda. Assuming a Susceptible-Infected-Susceptible model, we show how the FOI relates to the point prevalence, enabling the estimation of the FOI by modelling the prevalence using a generalized linear mixed model. We derive bounds for varying parasite clearance distributions. The resulting FOI varies significantly with age and is estimated to be highest among children aged 5-10 years in areas of high and medium malaria transmission and highest in children aged below 1 year in a low transmission setting. Heterogeneity is greater between than within households and it increases with decreasing risk of malaria infection. This suggests that next to the individual's age, heterogeneity in malaria FOI may be attributed to household conditions. When estimating the FOI, accounting for both observed and unobserved heterogeneity in malaria acquisition is important for refining malaria spread models.

Key words: Clearance rate distribution, generalized linear mixed model, point prevalence, SIS compartmental model.

\section{INTRODUCTION}

Estimating the burden of malaria and evaluating the impact of control strategies, requires reliable estimates of transmission intensities [1]. Measures of malaria transmission intensity include the entomological inoculation rate (EIR), parasite prevalence and force

\footnotetext{
* Author for correspondence: L. Mugenyi, Hasselt University, Agoralaan 1, 3590 Diepenbeek, Belgium.

(Email: levicatus.mugenyi@uhasselt.be)
}

of infection (FOI) [1-6]. The EIR is defined as the number of infectious bites per person per unit time $[2,7]$, whereas the FOI is defined as the number of infections per person per unit time [4] or the per capita rate at which a susceptible individual acquires infection $[8,9]$. The malaria FOI counts all incident (i.e., new) human malaria infections in a specified time interval regardless of clinical symptoms and recurrent infections [4]. The EIR and FOI are related but differ; the EIR considers the number of infective bites delivered by the mosquito vector, whereas the FOI focuses 
on the infections acquired by the human host. In theory, there should be close relationship between the EIR and the FOI, especially in children with less developed immunity. In practice, however, there is a discrepancy between the two because not every infectious bite results in an infection due to various factors [10]. The efficiency of transmission can be estimated by taking the ratio of the two measures, i.e., the ratio of the EIR to the FOI, the number of infectious bites required to cause an infection [10]. A smaller ratio of the EIR to the FOI implies higher transmission efficiency. Most studies have shown that malaria transmission is highly inefficient [4]; whereas more recently malaria FOI has been estimated from serological data $[1,11]$ by detecting past exposure to malaria infection, here we focus on estimating malaria FOI from parasitaemia data [12-14].

Despite well-recognized heterogeneity in malaria transmission [15, 16], the FOI is often estimated ignoring intrinsic variability in the individual risk of malaria infection. Heterogeneity in malaria infection arises due to variability in risk factors, including environmental, vector and host-related factors [17]. Taking these sources of heterogeneity into account $[15,17]$ in population-based epidemiological studies has been shown to be important [8].

Ronald Ross first published a mathematical model for malaria transmission in 1908 [16, 18]. This model was only firmly established in 1950 by the work of George Macdonald who used Ross's idea [16]. The 'Ross-Macdonald' model describes a simplified set of concepts that serves as a basis for studying mosquito-borne pathogen transmission [16]. Using this concept, mathematical methods to estimate the FOI in relation to the EIR have been proposed by, e.g., Smith et al. [3, 4], Keeling and Rohani [19] and Aguas et al. [20]. Some of the parameters involved in these models are often unknown and should be estimated from data [21]. A solution proposed by Ross in 1916 is to iterate between two modelling frameworks, that is, mathematical and statistical models [21, 22]. The major difference in these two is that the mathematical models (priori) are based on differential equations describing the biological mechanism and causal pathway of transmission, whereas the statistical models (posteriori) start by the statistical analysis of observations and work backwards to the underlying cause [21]. These two frameworks complement each other and, here, we provide an explicit link between them.

In this paper, we use the well-known generalized linear mixed model (GLMM) framework (see, e.g.,
[19]) to estimate the point prevalence accounting for both observed and unobserved heterogeneity and show how the FOI can be obtained from the point prevalence based on a mathematical SusceptibleInfected-Susceptible (SIS) model. We derive an expression and easy-to-calculate bounds of the FOI for varying parasite clearance distributions. Our results can be used to refine mathematical malaria transmission models.

\section{METHODS}

\section{Source of data}

The results in this paper are based on cohort data from children aged $0 \cdot 5-10$ years in three regions in Uganda: Nagongera sub-county, Tororo district; Kihihi sub-county, Kanungu district; and Walukuba sub-county, Jinja district. The data were collected as part of the Program for Resistance, Immunology, Surveillance and Modelling of malaria (PRISM) study. The study regions are characterized by distinct transmission intensities. The EIR was previously estimated to be 310,32 and $2 \cdot 8$ infectious bites per unit year, respectively, for Nagongera, Kihihi and Walukuba [6]. The study participants were recruited from 300 randomly selected households (100 per region) located within the catchment areas. Data were routinely collected every 3 months (routine visits) and for non-routine clinical (symptomatic) visits. Individuals were tested for the presence of Plasmodium parasites using microscopy from August 2011 to August 2014 (3 years). All symptomatic malaria infections were treated with artemether-lumefantrine (AL) anti-malarial medications. More detailed information regarding the study design can be found in Kamya et al. [6]. Given that for clinical visits the sampling process is outcome dependent (see the 'Discussion' section), the analysis here is restricted to the planned routine visits yielding unbiased estimates (simulation study, not shown).

\section{The SIS model, point prevalence and FOI}

A simplified version of malaria transmission can be described using the so-called SIS compartmental transmission model. This mathematical model classifies the population into two compartments, i.e., the susceptible $(S)$ and the infected $(I)$ class, which can be graphically depicted as shown in Figure 1. 


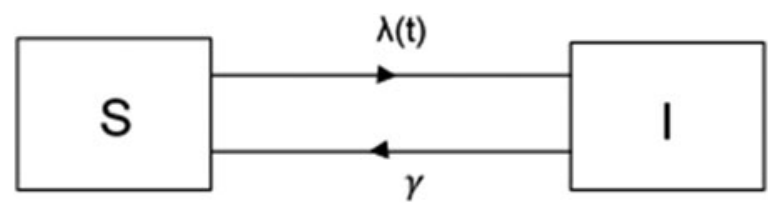

Fig. 1. A schematic diagram of the SIS compartmental model illustrating the simplified dynamics for malaria transmission.

Here, the rate $\lambda(t)$ at which individuals leave the susceptible state $S$ at time $t$ and flow to the infected state $I$, as they are infected with malaria parasites, is referred to as the FOI. Furthermore, $\gamma$ represents the time-invariant clearance rate at which individuals regain susceptibility after clearing malaria parasites from their blood. Let $s(t)$ denote the proportion of susceptible individuals in the population and $i(t)$ the proportion of infected individuals at calendar time $t$, i.e., the (point) prevalence, then the following set of ODEs (ordinary differential equations) describes transitions in the compartmental SIS model:

$\left\{\begin{array}{c}s^{\prime}(t)=-\lambda(t) s(t)+\gamma i(t), \\ i^{\prime}(t)=\lambda(t) s(t)-\gamma i(t) .\end{array}\right.$

As individuals are either susceptible to infection or malaria infected (at least in the aforementioned simplified SIS model), we have $s(t)=1-i(t)$. Substituting this expression for $s(t)$ into (1) yields:

$\lambda(\mathrm{t})=\frac{i(t) \gamma+i^{\prime}(t)}{1-i(t)}$,

where $i^{\prime}(t)$ is the derivative of the point prevalence with respect to $t$. The FOI $\lambda(t)$ can thus be estimated using an estimate for the prevalence $i(t)$ and the clearance rate $\gamma$.

Relaxing the assumption of an exponentially distributed parasite clearance distribution in the SIS model can be done by dividing the $I$ compartment into $J$ sub-compartments, such that infected individuals move from the first sub-compartment $I_{1}$ to the second $I_{2}$, and later to the $J^{\text {th }}$ sub-compartment $I_{J}$ during the different phases of clearing malaria parasites. Using identical rates $\gamma$ for the transitions between these sub-compartments and for moving from $I_{J}$ back to the $S$ compartment results in an Erlang distribution with shape parameter $J$ and rate $\gamma$ for the time spent in all of the sub-compartments [23]. It is easily shown that equation (2) yields an upper bound for the FOI when compared with the aforementioned Erlang clearance distribution (see the Appendix). A lower bound is readily obtained by taking $\gamma=0$ in equation (2) (SI model - see the Appendix). The FOI is thus bounded by $\left[\lambda_{L}(t), \lambda_{U}(t)\right]=\left[i^{\prime}(t) /(1-i(t))\right.$, $\left.\left.\left(\left(i^{\prime} t\right)+\gamma i(t)\right) /(1-i(t))\right)\right]$.

Estimates for both the exponential assumption (upper bound) as well as the lower bound are presented in this paper. In order to estimate the prevalence $\pi(t) \equiv$ $i(t)$, we use a GLMM to account for individual- and household-specific clustering. This will enable us to explicitly model the observed and unobserved heterogeneity in the acquisition of malaria infection.

\section{Generalized linear mixed model}

GLMMs extend the well-known generalized linear models by explicitly taking into account (multiple levels of) clustering of observations [24].

Let $Y_{i j k}$ denote the binary response variable indicating parasitaemia in the blood ( 1 if parasites are present - malaria infected; and 0 if not - malaria uninfected) for the $i^{\text {th }}$ individual nested in the $J^{\text {th }}$ household at the $k^{\text {th }}$ visit. Similarly, let $X_{i j k}$ be a $(p+1) \times 1$ vector containing covariate information on $p$ independent variables, and $Z_{i j k}$ be a $q \times 1$ vector of information associated with $q$ random effects. Given the subject-specific random effects $\boldsymbol{b}_{i j}$ and the covariate information $X_{i j k}$, the random variables $Y_{i j k} \mid X_{i j k}$ are assumed to be conditionally independent with conditional mean $\pi\left(X_{i j k} \mid \boldsymbol{b}_{i j}\right)=E\left(Y_{i j k} \mid X_{i j k}, \boldsymbol{b}_{i j}\right)=P$ $\left(Y_{i j k}=1 \mid X_{i j k}, \boldsymbol{b}_{i j}\right)$. The GLMM relates the conditional mean to the covariates $X_{i j k}$ and $Z_{i j k}$ as follows:

$$
\begin{aligned}
g\left[\pi\left(X_{i j k} \mid \boldsymbol{b}_{i j}\right)\right] & =g\left[P\left(Y_{i j k}=1 \mid X_{i j k}, \quad \boldsymbol{b}_{i j}\right)\right] \\
& =X_{i j k}^{T} \boldsymbol{\beta}+Z_{i j k}^{T} \boldsymbol{b}_{i j} .
\end{aligned}
$$

Here, $g$ is a monotonic link function (e.g., logit, cloglog and $\log ) ; \eta\left(X_{i j k} \mid \boldsymbol{b}_{i j}\right)=X_{i j k}^{T} \boldsymbol{\beta}+Z_{i j k}^{T} \boldsymbol{b}_{i j}$ is the linear predictor with $\boldsymbol{\beta}$ a vector of unknown regression parameters for the fixed effects; $\boldsymbol{b}_{i j} \sim N(0, \mathbf{D})$ a vector of subject-specific random effects for subject $i$ in household $j$ for which elements are assumed to be mutually independent; and D a $q \times q$ variance-covariance matrix [25].

Using equations (2) and (3), the FOI can be obtained using different link functions. Table 1 presents the prevalence and FOI when selecting either the logit, cloglog or log-link function in the GLMM.

\section{Flexible parametric modelling}

In a parametric framework such as the GLMM, fractional polynomials provide a very flexible modelling 
Table 1. General structures for the FOI according to different link functions in a GLMM framework

\begin{tabular}{lll}
\hline \hline Link function $(g)$ & Prevalence $(\pi)$ & FOI $(\lambda)$ \\
\hline Logit & $\frac{e^{\eta}}{1+e^{\eta}}$ & $\gamma e^{\eta}+\eta^{\prime} \frac{e^{\eta}}{1+e^{\eta}}$ \\
Cloglog & $1-e^{-e^{\eta}}$ & $\gamma\left(e^{e^{\eta}}-1\right)+\eta^{\prime} e^{\eta}$ \\
Log & $1-e^{-\eta}$ & $\gamma\left(e^{\eta}-1\right)+\eta^{\prime}$ \\
\hline \hline
\end{tabular}

$\eta$ refers to the linear predictor $\eta\left(X_{i j k} \mid \boldsymbol{b}_{i j}\right)$ and $\eta^{\prime}$ represents the derivative of the linear predictor with respect to the predictor of interest.

tool for the linear predictor $\eta\left(X_{i j k} \mid \boldsymbol{b}_{i j}\right)[21,26,27]$. In this paper, a GLMM using a fractional polynomial of degree one with regard to age, with power $p$ selected from a grid $(-3,-2,-1,-0 \cdot 5,0,0 \cdot 5,1,2,3)$ using Akaike's information criterion (AIC), is used [28]. More precisely, we use

$$
\begin{aligned}
\eta\left(X_{i j k} \mid \boldsymbol{b}_{i j}\right)= & \eta\left(a_{i j k}, l_{i j} \mid \boldsymbol{b}_{i j}\right) \\
= & \beta_{0}+\beta_{1} \operatorname{age}_{i j k}^{p}+\beta_{2} l_{i j}+b_{0 i(j)} \\
& +b_{1 i(j)} \operatorname{age}_{i j k}^{p},
\end{aligned}
$$

where $b_{0 i(j)}$ is the nested random intercept and $b_{1 i(j)}$ is the nested random slope for age. Nesting is done to explicitly acknowledge that individuals make up households. Furthermore, shifted year of birth: $l_{i j}$, defined as the child's birth year minus the birth year of the oldest child in the cohort (i.e., baseline year 2001), is used in the model to account for the (calendar) time effect since $[$ calendar time $]=[$ birth year $]+$ [age]. The linear predictor (4) can be further extended to include additional covariates.

\section{Age-time-dependent FOI}

In equation (3), the conditional mean $\pi\left(X_{i j k} \mid \boldsymbol{b}_{i j}\right)$ is the point prevalence conditional on the random and fixed effects. In this paper, we use the logit-link function, which enables easy calculation of the ICC (intracluster correlation coefficient) through an approximation indicating how much the elements within a cluster are correlated [24, 29, 30].

The age-time-dependent FOI, conditional on random effects, is estimated by plugging in the parameter estimates obtained from the final fit in equation (2). More specifically, using a logit-link, the conditional age-time-dependent FOI is estimated as follows:

$$
\begin{aligned}
\hat{\lambda}_{l i j}\left(a_{i j k} \mid \boldsymbol{b}_{i j}\right)= & \hat{\gamma} e^{\hat{\eta}\left(a_{i j k}, l_{i j} \mid \boldsymbol{b}_{i j}\right)} \\
& +\hat{\eta}^{\prime}\left(a_{i j k}, l_{i j} \mid \boldsymbol{b}_{i j}\right) \hat{\pi}_{l i j}\left(a_{i j k}, l_{i j} \mid \boldsymbol{b}_{i j}\right)
\end{aligned}
$$

where $\hat{\gamma}$ is an estimate for the clearance rate and $\hat{\pi}_{l i j}\left(a_{i j k}, l_{i j} \mid \boldsymbol{b}_{i j}\right)$ is the estimated age- and timedependent conditional prevalence. For the lower boundary of FOI, $\hat{\gamma} e^{\hat{\eta}\left(a_{i j k}, l_{i j} \mid \boldsymbol{b}_{i j}\right)}$ is omitted in equation (5). In the above expression, an estimate for the clearance rate $\gamma$ is required. Previously, Bekessy et al. [12] estimated annual clearance rates of $1.643,0.584$ and 0.986 years $^{-1}$ for children aged $<1,1-4$ and 5-8 years, respectively. Later, Singer et al. [14] estimated these rates as $1.917,1.425$ and 2.364 years $^{-1}$ for ages $<1,1-4$ and 5-8 years, respectively. Sama et al. [13] estimated a constant annual clearance rate of 1.825 years ${ }^{-1}$ by assuming an exponential distribution for infection duration or parasite clearance. Most recently, Bretscher et al. [31] studied the parametric distributions of the infection durations using Ghanaian data, and concluded based on AIC that a Weibull distribution gave a better fit to the data followed by a gamma distribution, while an exponential one was performing worst. In this paper, we use both exponential and Erlang clearance distributions to derive estimates for the malaria FOI obtained based on the aforementioned clearance rates as distributional parameters.

Often, an investigator may wish to observe population averaged estimates. Under the random effects framework, this can be achieved by taking the expectation of the conditional estimates (e.g., the FOI in (5)) resulting into unconditional or marginal estimates. Using the logit-link function, the unconditional (population) FOI is given by

$$
\begin{aligned}
\lambda_{l i j}\left(a_{i j k}\right)= & E\left(\lambda_{l i j}\left(a_{i j k} \mid \boldsymbol{b}_{i j}\right)\right) \\
= & E\left(\gamma e^{\eta\left(a_{i j k}, l_{i j} \mid \boldsymbol{b}_{i j}\right)}\right. \\
& \left.+\eta^{\prime}\left(a_{i j k}, l_{i j} \mid \boldsymbol{b}_{i j}\right)^{*} \pi_{l i j}\left(a_{i j k}, l_{i j} \mid \boldsymbol{b}_{i j}\right)\right) .
\end{aligned}
$$

Calculation of the marginalized FOI in (6), requires integrating out the random effects, $\boldsymbol{b}_{i j}$, over their fitted distribution. This can be done using numerical integration techniques or based on numerical averaging [24].

\section{Model selection}

Model building was done using both AIC [32] and a likelihood ratio test for the random effects based on the appropriate mixture of chi-square distributions [33]. Backward model building was performed starting with the random effects and then the fixed effects. The covariates considered in the model-building process included study site, age, time since enrolment, shifted 
birth year (i.e., shifted birth year $=$ birth year-birth year of the oldest child), previous use of AL treatment and the infectious status at the previous visit. The covariates, 'time since enrolment' and 'shifted birth year' were generated to represent the calendar time, albeit we preferred the latter one since participants were not enrolled at the same time point.

\section{RESULTS}

Of 989 children, recruited between August 2011 and August 2014, $334(33.8 \%), 355(35.9 \%)$ and $300(30 \cdot 3 \%)$ were from Nagongera, Kihihi and Walukuba, respectively. The baseline parasite prevalence among children aged below 5 years was $38 \cdot 2 \%$, $12 \cdot 8 \%$ and $9 \cdot 5 \%$ for Nagongera, Kihihi and Walukuba, respectively. The monthly parasite prevalence was higher in Nagongera (range: 26.7-68.4\%) followed by Kihihi (range: $7 \cdot 0-68 \cdot 0 \%$ ) and lastly by Walukuba (range: $0-42 \cdot 9 \%$ ). Other summary statistics are presented in Table 2. In general, the prevalence was higher among older children (5-10 years).

The parasite prevalence increases with age particularly for children $<3$ years of age and after 7 years of age a decrease is observed (Fig. 2, panel A). The prevalence increases with calendar time in Kihihi with increasing variability, while it decreases in Walukuba, and slightly increases in Nagongera (Fig. 2, panel B). These observations suggest a difference in malaria infection risk between the three study sites. Also, the infection risk seems to vary with age and calendar time and it tends to take different trends between sites indicating a possibility for a site-time interaction effect. The relationship with age seems to be non-linear. These observed effects were taken into consideration when building the GLMM.

The mean structure in our model consists of a fractional polynomial of age with power -1 (selected based on AIC) and the following covariates (based on significance testing at 5\% significance level): shifted year of birth; infection status at previous visit and $\mathrm{AL}$ use; and study site. Goodness-of-fit of the final model was assessed using the ratio of the generalized Chi-square statistic to its degrees of freedom. A value of 0.74 was obtained, which is fairly close to 1 , indicating that the variability in these data seems to be adequately modelled and little residual overdispersion remains present [34].

The parameter estimates, standard errors, and corresponding test results of the final GLMM fit are shown in Table 3. More details about the candidate models can be found in the Appendix (Tables A1 and A2) together with the fitted conditional and marginal prevalences for the different AL use categories (Fig. A2). The results in Table 3 show an overall significant effect of age and shifted year of birth; the effect of age and shifted year of birth is non-significant and borderline significant, respectively, for Walukuba, whereas the effect of age is significant for Kihihi and Nagongera. Shifted year of birth is significant for Kihihi and non-significant for Nangongera. There is significant heterogeneity in the rate of acquiring malaria infection between households (Walukuba: variance $=2 \cdot 80 ; \quad$ Kihihi: variance $=1 \cdot 16 ; \quad$ Nagongera: variance $=0 \cdot 21)$ and between household members (variance $=0 \cdot 24$ ). The intra-household correlation coefficients are $0.44,0.25$ and 0.06 for Walukuba, Kihihi and Nagongera, indicating moderate, low and very low correlation within households, respectively. The intra-individual correlation coefficients are $0.04,0.05$ and 0.06 for Walukuba, Kihihi and Nagongera, respectively, indicating very low correlation in all sites.

Based on the final model fit and using equations (5) and (6) both the conditional (given the random effects) and marginal (population averaged) FOIs can be calculated provided that $\gamma$ can be estimated. However, estimating $\gamma$ from the same data is not possible due to an identifiability problem: two or more distinct values of $\gamma$ give rise to the same (log)likelihood (see Fig. A1 in the Appendix). Therefore, we use $\gamma$ equal to the annual clearance rates given by Bekessy et al. [12] as 1.643, 0.584 and 0.986 years ${ }^{-1}$ for children aged $<1,1-4$ and 5-10 years, respectively, to calculate the conditional and marginal FOIs. We further conduct a sensitivity analysis by considering different clearance rates ranging from 0 to 3 motivated by the ranges estimated by Bekessy et al. [12], Singer et al. [14], Sama et al. [13] and Bretscher et al. [31] (see Fig. 5, top row). As discussed before, we also provide lower bounds for the FOI.

Figure 3 shows estimates for the marginal FOI together with the corresponding lower bound estimates. We focused on children who were born in the baseline year for graphical reasons. Similar plots were obtained (not shown) for other birth years. Estimates for the lower boundary of the FOI were higher in Nagongera followed by Kihihi and Walukuba. For Nagongera and Walukuba, the lower bound for the FOI was highest for children aged below 1 year and least in those aged 5-10 years, yet. In Kihihi, it is highest among those aged 1-4 years. 
Table 2. Recruited number of children, baseline and monthly parasite prevalence, by study site and age group

\begin{tabular}{|c|c|c|c|c|}
\hline & & Nagongera & Kihihi & Walukuba \\
\hline \multirow[t]{3}{*}{$<5$ years } & Number & 186 & 188 & 190 \\
\hline & Baseline prevalence $^{\mathrm{a}}(\%)$ & $38 \cdot 2$ & $12 \cdot 8$ & $9 \cdot 5$ \\
\hline & Monthly prevalence ${ }^{\mathrm{a}}(\%)$, range & $27 \cdot 4-54 \cdot 7$ & $7 \cdot 0-64 \cdot 7$ & $0-32 \cdot 0$ \\
\hline \multirow[t]{3}{*}{$5-10$ years } & Number & 148 & 167 & 110 \\
\hline & Baseline prevalence ${ }^{\mathrm{a}}(\%)$ & $58 \cdot 8$ & $18 \cdot 0$ & $10 \cdot 9$ \\
\hline & Monthly prevalence ${ }^{\mathrm{a}}(\%)$, range & $26 \cdot 7-68 \cdot 4$ & $8 \cdot 3-68 \cdot 0$ & $0-42 \cdot 9$ \\
\hline \multirow[t]{3}{*}{ Total } & Number & 334 & 355 & 300 \\
\hline & Baseline prevalence $^{\mathrm{a}}(\%)$ & $47 \cdot 3$ & $15 \cdot 2$ & $10 \cdot 0$ \\
\hline & Monthly prevalence ${ }^{\mathrm{a}}(\%)$, range & $26 \cdot 7-68 \cdot 4$ & $7 \cdot 0-68 \cdot 0$ & $0-42 \cdot 9$ \\
\hline
\end{tabular}

a Parasite prevalence.
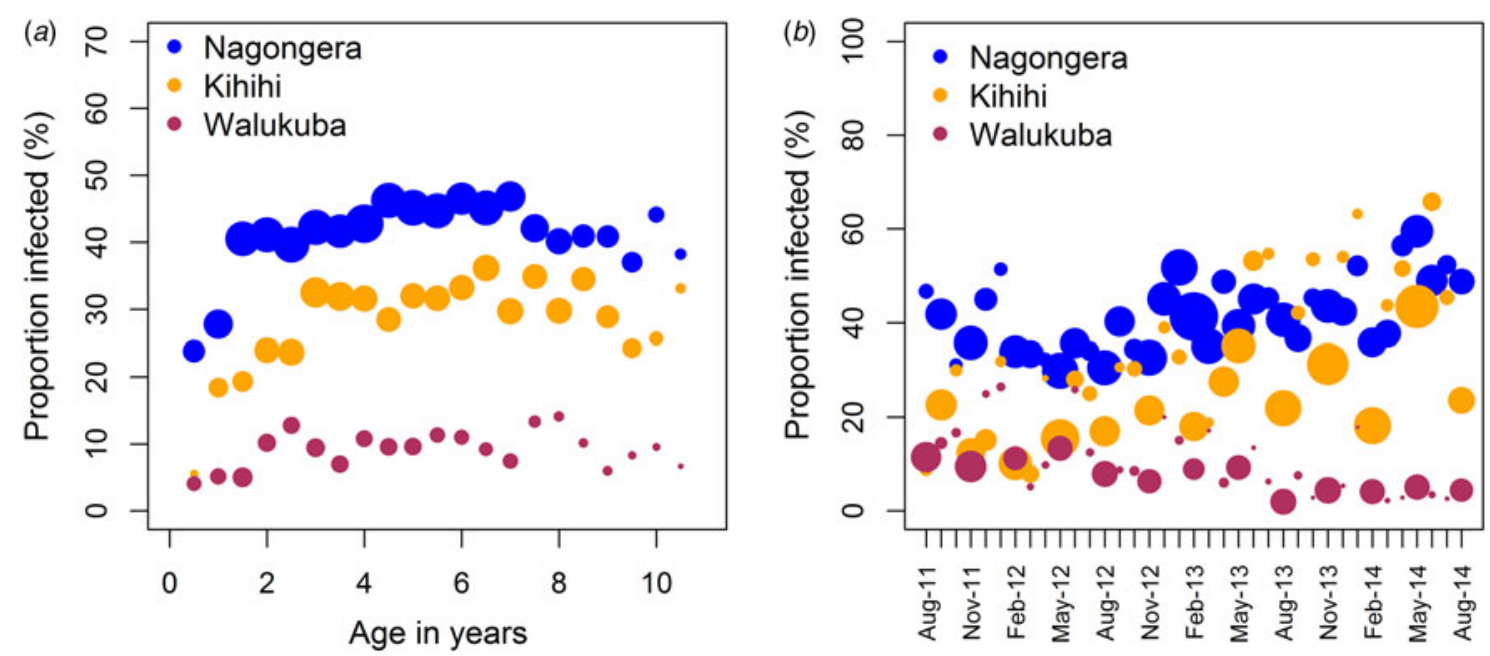

Fig. 2. Proportion of children infected with malaria parasites (parasitaemia) in a cohort followed for 3 years, by study site (Nagongera, Kihihi and Walukuba) in Uganda based on data from August 2011 to August 2014 with the size of the dots proportional to the number of observations. (A) Observed parasitaemia varying with age; (B) observed parasitaemia varying with calendar time.

Figure 3 further shows that in Nagongera and Kihihi, the estimates for the marginal FOI were highest among children aged 5-10 years; yet in Walukuba it was highest among those aged below 1 year. The values for the marginal FOI obtained using the upper boundary estimator, stratified by site, age group and the previous infection status and use of $\mathrm{AL}$ are given in Table A3 in the Appendix. At the extreme, the previously symptomatic children acquire up to four infections per year in Nagongera, and eight infections per year both in Kihihi and Walukuba. Overall, the FOI is highest among the asymptomatic children and smallest among previously symptomatic children across all age groups and sites (Fig. 3 and Table 3A). Although Figure 3 clearly shows the impact of different distributional assumptions with regard to the clearance time, the lower and upper bound estimates do not fully capture uncertainty around the point estimates. In Table A4 of the Appendix, we show the $95 \%$ confidence bounds for the age- and time-dependent FOI.

Figure 4 (top row) shows the predicted conditional FOIs for 50 randomly selected individual profiles at each of the three sites based on the lower boundary estimator for the FOI. For graphical purposes, we focused on subjects who were symptomatic at the previous visit and who were born in the baseline year. However, similar plots are obtained for other levels of the infection status at the previous visit and for different birth years. Figure 4 (bottom row) shows the predicted marginal FOIs again based on the lower boundary estimator, by age (continuous scale) and infection status at the previous visit and past $\mathrm{AL}$ use. In general, the lower boundary estimator 
Table 3. Estimates of the fitted GLMM using a fractional polynomial of degree 1 for age and a logit-link function

\begin{tabular}{|c|c|c|c|c|c|c|}
\hline Effect & & Parameter & $\begin{array}{l}\log \text { OR } \\
\text { (s.E.) }\end{array}$ & $t$-value & $P$ & OR \\
\hline Intercept & & $\beta_{0}$ & $-3 \cdot 04(0 \cdot 38)$ & $-8 \cdot 09$ & $<0.001$ & \\
\hline \multirow[t]{2}{*}{ Study site $($ Reference $=$ Walukuba $)$} & Kihihi & $\beta_{1}$ & $0 \cdot 86(0 \cdot 43)$ & $2 \cdot 01$ & $0 \cdot 045$ & $2 \cdot 36(1 \cdot 02-5 \cdot 49)$ \\
\hline & Nagongera & $\beta_{2}$ & $2 \cdot 19(0 \cdot 40)$ & $5 \cdot 45$ & $<0 \cdot 001$ & $8 \cdot 94(4 \cdot 08-19 \cdot 57)$ \\
\hline \multirow[t]{3}{*}{$\begin{array}{l}\text { Infection status at the previous visit ( } \mathrm{Ref}= \\
\text { Negative and No AL treatment in past) }\end{array}$} & $\begin{array}{l}\text { Negative + } \\
\text { AL }\end{array}$ & $\beta_{3}$ & $-0 \cdot 01(0 \cdot 10)$ & $-0 \cdot 05$ & $0 \cdot 956$ & $0 \cdot 99(0 \cdot 82-1 \cdot 21)$ \\
\hline & Symptomatic & $\beta_{4}$ & $-0 \cdot 24(0 \cdot 10)$ & $-2 \cdot 30$ & $0 \cdot 022$ & $0 \cdot 78(0 \cdot 64-0.97)$ \\
\hline & Asymptomatic & $\beta_{5}$ & $1 \cdot 23(0 \cdot 12)$ & $9 \cdot 94$ & $<0.001$ & $3 \cdot 43(2 \cdot 69-4 \cdot 37)$ \\
\hline \multirow[t]{3}{*}{$\mathrm{Age}^{-1}$} & Walukuba & $\beta_{6}$ & $-0 \cdot 05(0 \cdot 83)$ & $-0 \cdot 06$ & $0 \cdot 948$ & $0.95(0 \cdot 19-4 \cdot 82)^{\mathrm{b}}$ \\
\hline & Kihihi & $\beta_{7}$ & $-4 \cdot 01(0 \cdot 87)$ & $-4 \cdot 62$ & $<0.001$ & $0.02(0 \cdot 003-0 \cdot 10)^{\mathrm{b}}$ \\
\hline & Nagongera & $\beta_{8}$ & $-1 \cdot 75(0 \cdot 45)$ & $-3 \cdot 89$ & $0 \cdot 001$ & $0 \cdot 17(0 \cdot 07-0 \cdot 42)^{\mathrm{b}}$ \\
\hline \multirow[t]{3}{*}{ Shifted year of birth ${ }^{\mathrm{a}}$} & Walukuba & $\beta_{9}$ & $-0 \cdot 13(0 \cdot 06)$ & $-2 \cdot 00$ & $0 \cdot 045$ & $0 \cdot 88(0 \cdot 78-1 \cdot 00)$ \\
\hline & Kihihi & $\beta_{10}$ & $0 \cdot 11(0 \cdot 04)$ & $2 \cdot 58$ & $0 \cdot 010$ & $1 \cdot 12(1 \cdot 13-1 \cdot 22)$ \\
\hline & Nagongera & $\beta_{11}$ & $0 \cdot 04(0 \cdot 03)$ & $1 \cdot 33$ & $0 \cdot 184$ & $1 \cdot 04(0 \cdot 98-1 \cdot 10)$ \\
\hline Variance components & & & Variance & $z$-value & & \\
\hline Variance for random intercepts for subjects & & $d_{11}$ & $0 \cdot 24(0 \cdot 07)$ & $3 \cdot 32$ & $<0 \cdot 001$ & \\
\hline \multirow[t]{3}{*}{ Variance for random intercepts for households } & Walukuba & $d_{22}$ & $2 \cdot 80(0 \cdot 88)$ & $3 \cdot 20$ & $0 \cdot 001$ & \\
\hline & Kihihi & $d_{33}$ & $1 \cdot 16(0 \cdot 28)$ & $4 \cdot 21$ & $<0.001$ & \\
\hline & Nagongera & $d_{44}$ & $0 \cdot 21(0 \cdot 08)$ & $2 \cdot 48$ & $0 \cdot 007$ & \\
\hline
\end{tabular}

${ }^{\text {a }}$ Birth year - min (birth year).

${ }^{\mathrm{b}}$ Note that the OR here should be interpreted at the Age $^{-1}$ level.

indicates that younger children have the greatest FOI. In all sites, individuals that were asymptomatic at the previous visit have the highest FOI, regardless of age. The depicted conditional FOI curves show that individuals have different profiles, indicating substantial unobserved heterogeneity. The increasing trend in the FOI from 6 months of age is likely attributed to loss of maternal immunity in infants [35].

Figure 5 (top row) shows the marginal FOIs for different clearance rates ranging from 0 up to 3 years $^{-1}$ ( $y$-axis). For graphical purposes, and without loss of generality, we again focused on subjects who were symptomatic at the previous visit and who were born in the baseline year. The colour gradient from green (dark) to brown (light) in Figure 5 (top row) corresponds to an increasing FOI. The figure indicates that in Nagongera and Kihihi, children who are below 1 year of age have a lower FOI (green colour) regardless of the presumed clearance rate. Also, in Nagongera and Kihihi, the risk for malaria infection increases with increasing clearance rate, except for the younger children $<1-2$ years. In Walukuba, the FOI increases with increasing clearance rate regardless of age.

Figure 5 (bottom row) shows how the FOI varied with age groups $(\mathrm{A} \leq 1$ year, $\mathrm{B}=1-4$ years, $\mathrm{C}=5$ 10 years) and calendar time among subjects assumed to be symptomatic at the previous visit. In Kihihi, the risk of acquiring a new malaria infection is slightly higher for children born in 2010 compared with those born in earlier years across age groups but not for Nagongera and Walukuba. This would be expected since children born at a later year are younger than those born at an earlier year, and hence are at a higher risk of infection.

\section{DISCUSSION}

In this paper, we use data from a cohort study to estimate the malaria FOI among Ugandan children while accounting for observed and unobserved heterogeneities. The results clearly demonstrate the existence of heterogeneity in the acquisition of malaria infections, which is greater between households than between household members. These observations emphasize the claim by White et al. [17] that heterogeneity in malaria infection can arise due to several unobserved factors, including environmental, vector and host-related factors. This implies that estimating the malaria transmission parameters assuming homogeneity in the acquisition of infection may yield misleading results.

The findings were based on the use of a readily available statistical method, the GLMM, which 


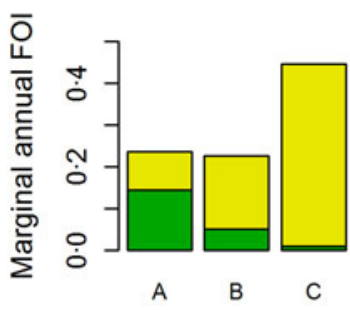

Negative, No AL

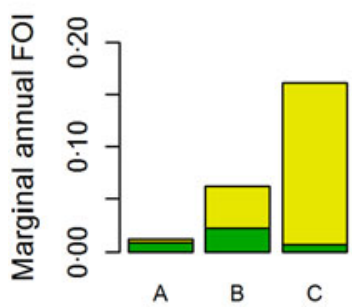

Negative, No AL

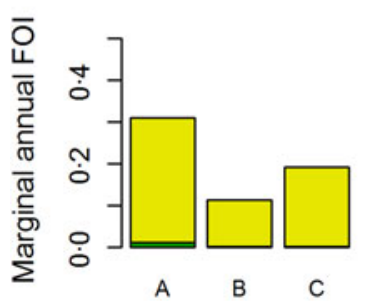

Negative, No AL
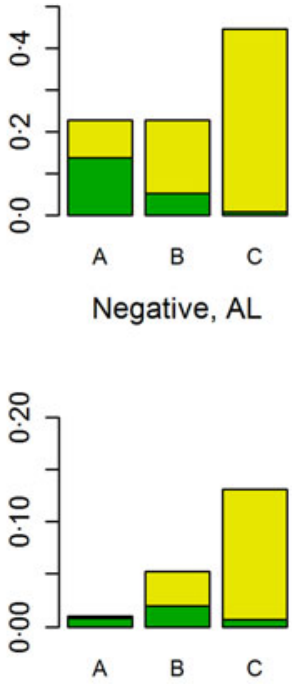

Negative, AL

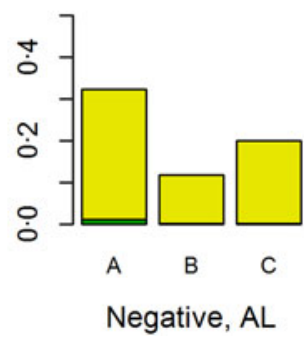

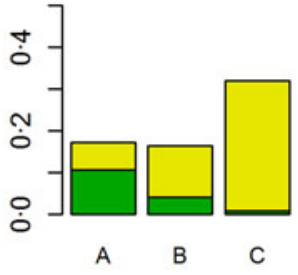

Symptomatic
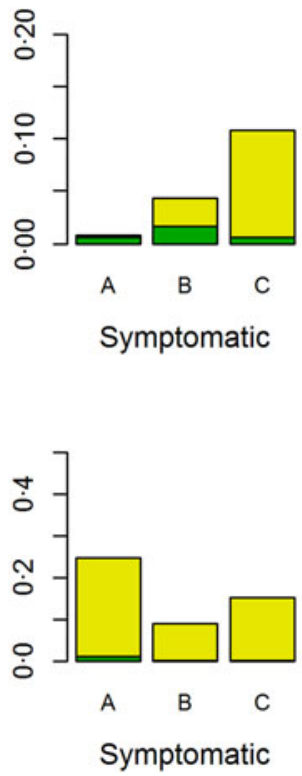

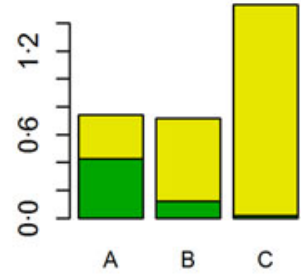

Asymptomatic

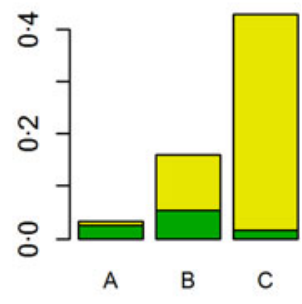

Asymptomatic

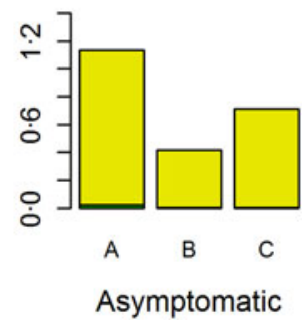

Fig. 3. The lower bound (green) for the marginal annual FOI and the difference between upper and lower bound (yellow) with full bar showing the upper bound for the FOI, by study site, age group (A, <1 year; B, 1-4 years; and C, 5-10 years) and the infection status at the previous visit and past use of AL (negative and no AL in the past (left column), negative and $\mathrm{AL}$ in the past (second left column), symptomatic (second right column) and asymptomatic (right column)) for children assumed to be born in the baseline year (2001). Top row, Nagongera; middle row, Kihihi; bottom row, Walukuba.

takes into account heterogeneity between individuals and households in the acquisition of malaria infection. In particular, a fractional polynomial of age of degree 1 and power -1 , adjusted for the calendar time, by means of the so-called 'shifted birth year' (i.e., shifted birth year = birth year-birth year of the oldest child), and other covariates, was considered. The fractional polynomial was chosen because it provides a very flexible modelling tool while retaining the strength of a parametric function. The random slope effects for the fractional polynomial function of age resulted in negative estimates for the FOI, which are biologically implausible and therefore the random slopes were dropped. This could be perceived as a drawback of using the GLMM in combination with fractional polynomials and a more mechanistic approach in which heterogeneity is taken into account at different levels could prove valuable here (further research).
When allowing for serial correlation in the model through the specification of an AR(1) correlation structure, the model failed to converge, indicating that too little information was available in the PRISM data to accommodate serial correlation, at least when assuming that the $\mathrm{AR}(1)$ assumption is appropriate. An in-depth investigation thereof is an interesting topic for further research.

Based on the SIS model, we derived an expression relating the FOI to the prevalence for infectious diseases such as malaria where we cannot assume lifelong immunity. This expression is an extension of the one proposed by Hens et al. [21] for a so-called SIR model assuming lifelong immunity after recovery, an assumption, which is untenable for malaria. A compartmental model, which can account for temporally recovery due to prior use of treatment (induced immunity) or due to previous exposure to infection 

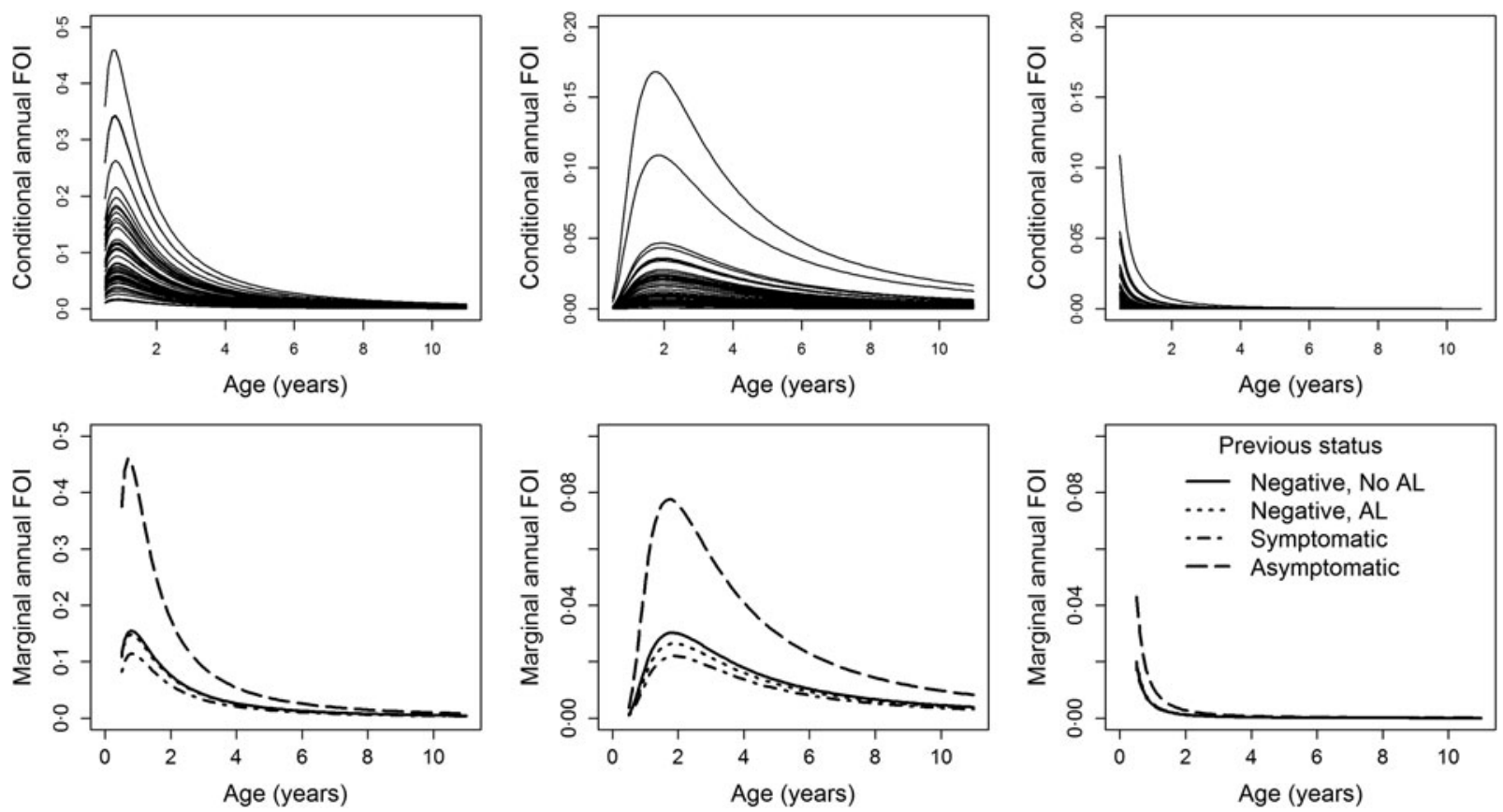

Fig. 4. Top row: Individual-specific evolutions for the conditional annual FOI obtained using the lower boundary estimator, by study site for children assumed to be symptomatic at the previous visit and who were born in the baseline year (2001). Bottom row: The marginal annual FOI, obtained using the lower boundary estimator, by study site and the infection status at the previous visit and past use of AL (negative and no AL in the past (solid lines), negative and AL in the past (dotted lines), symptomatic (dash-dotted lines) and asymptomatic (long-dashed lines)). Left column, Nagongera; middle column, Kihihi; right column, Walukuba.
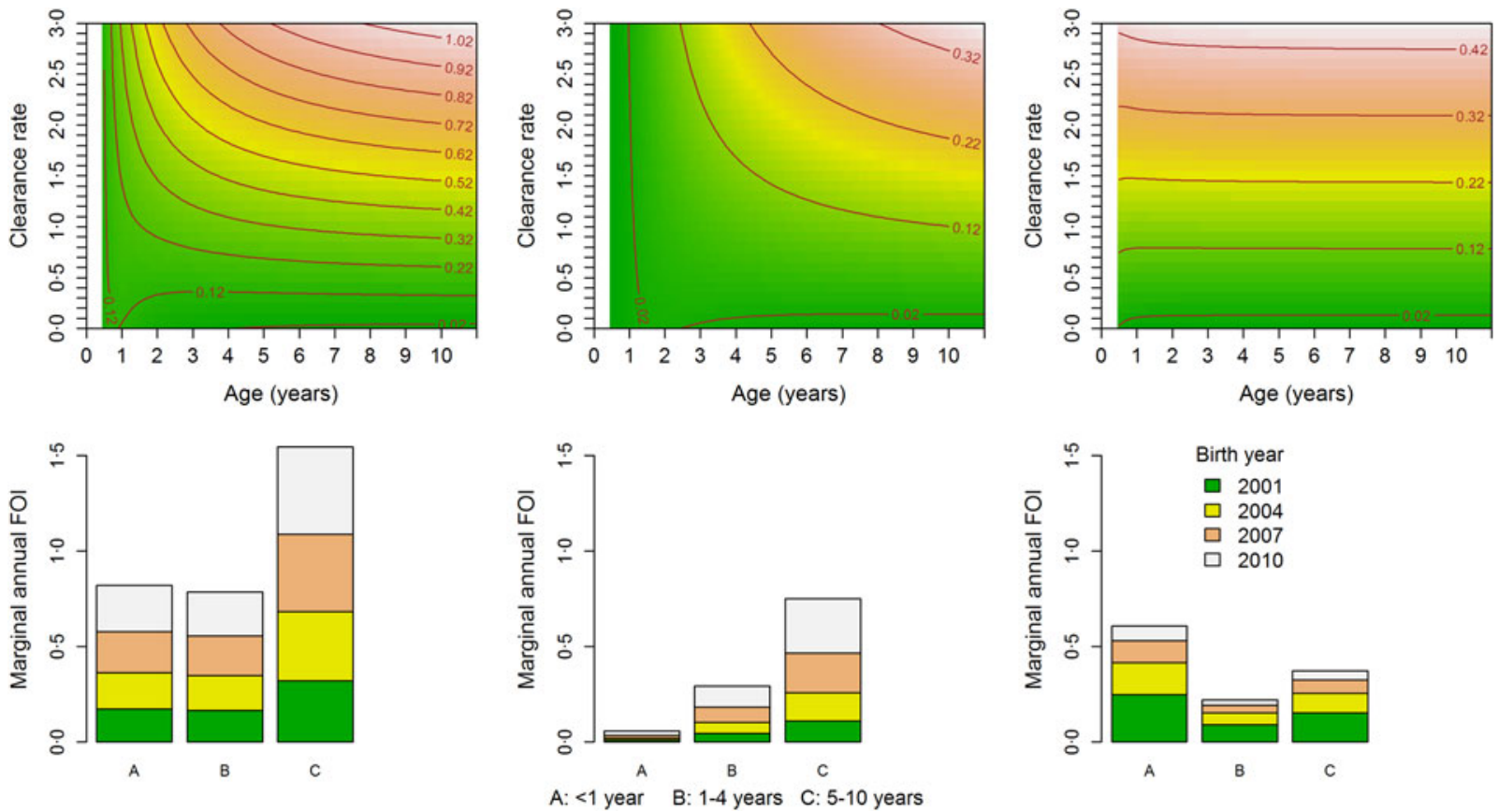

Fig. 5. Top row: The marginal annual FOI (contour lines) considering different values for the clearance rate ranging from 0 to 3 years $^{-1}$ by study site for individuals assumed to be symptomatic at the previous visit and were born in the baseline year. Bottom row: The marginal annual FOI, obtained using the upper boundary estimator, for individuals assumed to be symptomatic at the previous visit, by study site, birth year (2001, 2004, 2007 and 2010) and by age group (A: <1 year, B: 1-4 years, and C: 5-10 years). Left panel, Nagongera; middle panel, Kihihi; right panel, Walukuba. 
(acquired immunity), that is, Susceptible-InfectedRecovered(Treatment)-Susceptible (SIR(T)S), would potentially offer a better alternative compared with the more restrictive SIS model. However, an SIR(T)S model does not yield a closed-form expression for the point prevalence, and hence, for the FOI. Nevertheless, the derivations are approximately valid for an SIR(T)S model with short recovery duration (derivations not included here). Consequently, we focused on the SIS model, albeit that we adjusted for the previous infection status and treatment in our model. The standard SIS compartmental model assumes that the clearance rate is exponentially distributed. We derived two estimators for the FOI, which provide a lower and upper boundary for the FOI based on different Erlang distributions for the clearance rate. The lower boundary approximately holds for a scenario in which the clearance rate is small compared with the FOI. Although mathematical models encompassing more complicated and more realistic transmission dynamics for malaria could be considered, we defer their treatment to future research in which we will combine Nonlinear Mixed Model (NLMM) methodology and numerical approaches for the estimation of the model parameters in the presence of unobserved heterogeneity.

The temporal inhomogeneity observed in the data is not in contradiction with the SIS model we used. Heterogeneity, age and temporal aspects are addressed in the GLMM, through the specification of random effects as well as age- and calendar time variables; whereas derivations from the SIS model under endemic equilibrium enable the estimation of the age- and time-dependent FOI from the estimated age- and time-dependent parasite prevalence. Furthermore, estimation of the reproduction number can be done when focusing on the underlying mechanistic modelling of the FOI. However, we deem this to be beyond the scope of this specific manuscript. Seasonality is not explicitly modelled here; however, inclusion of a covariate describing the amount of rainfall, due to the absence of a clear distinction between the different seasons, and based on additional information (not part of the PRISM data) would be an interesting topic for further research.

When the clearance rate is considered negligible, the rate at which children get infected is highest among those between 1 and 2 years. When the clearance rate is non-negligible, the infection rate is higher among children older than 5 years in areas with high and medium transmission (e.g., Nagongera and
Kihihi) and higher in children below 1 year in areas with low transmission (e.g., Walukuba). In Kihihi, the FOI was least for children aged $<1$ year and it is observed to increase as children grow up from 6 months to 1 year. This could be explained by the fact that children lose maternal immunity in their first year of life [35], which puts them at an increased risk of malaria infection. The higher FOI among children aged 5 years and older could be explained by the fact that these children are often asymptomatic malaria cases and are rarely treated, which makes them reservoirs for infections. This finding conquers with the work by Walldorf et al. [36] who reported that children aged 6-15 years were at higher risk of (asymptomatic) infection compared with the younger ones. They concluded that older children represent an underappreciated reservoir of malaria infection and have less exposure to antimalarial interventions.

A higher risk was seen among children in Nagongera compared with those in Kihihi and Walukuba with no significant difference between the latter two sites. This could be explained by the fact that Nagongera is a predominantly rural area with many semi-structured houses and many mosquitoes compared with Walukuba or Kihihi as was noted by Kilama et al. [5]. Our results also demonstrated the importance of prior treatment in lowering infection risk due to the post-treatment prophylactic effect of longer acting anti-malarials, such as AL. For example, children who were previously treated with $\mathrm{AL}$ (the symptomatic malaria cases) had a lower risk of getting re-infected compared with those who were asymptomatic or negative at the previous visit.

This study has two major limitations. First, the analysis was based on results of parasite prevalence determined by microscopy, which is less sensitive than molecular methods such as PCR (polymerase chain reaction) or LAMP (loop-mediated isothermal amplification method) [37, 38]. Thus, sub-microscopic infections would not have been detected. This could have resulted into lower estimates of the FOI. In addition, genotyping was not performed to distinguish new and recurrent infections. As a result, the FOI among individuals who were asymptomatic at the previous visit could have been overestimated. Secondly, the unscheduled clinical visits by the symptomatic individuals were triggered by the study outcome (i.e., parasitaemia). This creates a dependency between the observation-time and outcome processes. This dependence, if not accounted for, has a potential to introduce bias in the model estimates and hence in 
the estimation of the FOI. This bias was avoided by dropping clinical visits and by using only routine data, although the infection status and use of treatment during clinical visits was accounted for in the model. This implies that the analysis used less data than was actually available. The latter limitation will be dealt with in future research by modelling both the outcome and the observation-time processes concurrently using a joint model $[39,40]$.

To conclude, we have used longitudinal data from a cohort of Ugandan children to estimate the malaria FOI accounting for both observed and unobserved heterogeneity. First, we show how the FOI relates to parasite prevalence assuming an SIS compartmental model and giving both lower and upper boundaries thereof by relaxing the exponential assumption with regard to the parasite clearance distribution. We estimated the parasite prevalence using a GLMM, whose estimates were used to obtain an estimate for the FOI. The malaria FOI was highest among children aged 1-2 years based on the lower boundary estimator, and it was higher among children older than 5 years in areas of high and medium transmission based on the upper boundary estimator. In a low transmission setting, the FOI was highest in children aged below 1 year regardless of the boundary estimator for the FOI. The FOI varied between study sites highest in Nagongera and least in Walukuba. Heterogeneity increases with decreasing FOI and is greater between households than household members. We recommend that estimating the malaria FOI should be done accounting for both observed and unobserved heterogeneity to enable refining existing mathematical models in which the FOI may be unknown.

\section{ACKNOWLEDGEMENTS}

Research reported in this publication was supported by the National Institute of Allergy and Infectious Diseases of the National Institutes of Health under Award Number U19AI089674. The content is solely the responsibility of the authors and does not necessarily represent the official views of the National Institutes of Health. The PRISM project gratefully acknowledges the Uganda Ministry of Health, our research team, collaborators and advisory boards, and especially all participants involved. The support from the PRISM team including Moses Kamya, Grant Dorsey, Sarah Staedke and Dave Smith is highly appreciated. Support from the IAP Research
Network P7/06 of the Belgian State (Belgian Science Policy) is gratefully acknowledged. This research was supported by the Antwerp Study Centre for Infectious Diseases. LM is supported by a grant of the Vlaamse Interuniversitaire Raad (VLIR). NH gratefully acknowledges support from the University of Antwerp scientific chair in Evidence-based Vaccinology, financed in 2009-2016 by a gift from Pfizer and in 2016 by GSK.

\section{DECLARATION OF INTEREST}

None.

\section{ETHICAL STANDARDS}

The authors assert that all procedures contributing to this work comply with the ethical standards of the relevant national and institutional committees on human experimentation and with the Helsinki Declaration of 1975 , as revised in 2008. The authors assert that all procedures contributing to this work comply with the ethical standards of the relevant national and institutional guides on the care and use of laboratory animals.

\section{REFERENCES}

1. Corran P, et al. Serology: a robust indicator of malaria transmission intensity. Trends in Parasitology 2007; 23: $575-582$.

2. Onori E, Grab B. Quantitative estimates of the evolution of a malaria epidemic in Turkey if remedial measures had not been applied. Bulletin of the World Health Organization 1980; 58: 321-326.

3. Smith DL, et al. The entomological inoculation rate and Plasmodium falciparum infection in African children. Nature 2005; 438: 492-495.

4. Smith DL, et al. A quantitative analysis of transmission efficiency versus intensity for malaria. Nature Communications 2010; 1: 108. doi:10.1038/ncomms1107.

5. Kilama M, et al. Estimating the annual entomological inoculation rate for Plasmodium falciparum transmitted by Anopheles gambiae s.l. using three sampling methods in three sites in Uganda. Malaria Journal 2014; 13: 111.

6. Kamya MR, et al. Malaria transmission, infection, and disease at three sites with varied transmission intensity in Uganda: implications for malaria control. American Journal of Tropical Medicine and Hygiene 2015; 92: 903-912.

7. Onori E, Grab B. Indicators for the forecasting of malaria epidemics. Bulletin of the World Health Organization 1980; 58: 91-98. 
8. Coutinho FAB, et al. Modelling heterogeneities in individual frailties in epidemic models. Mathematical and Computer Modelling 1999; 30: 97-115.

9. Hens $\mathbf{N}$, et al. Seventy-five years of estimating the force of infection from current status data. Epidemiology \& Infection 2010; 138: 802-812.

10. Smith DL, McKenzie FE. Statics and dynamics of malaria infection in Anopheles mosquitoes. Malaria Journal 2004; 3: 13.

11. Von Fricken ME, et al. Age-specific malaria seroprevalence rates: a cross-sectional analysis of malaria transmission in the Ouest and Sud-Est departments of Haiti. Malaria Journal 2014; 13: 361.

12. Bekessy A, Molineaux L, Storey J. Estimation of incidence and recovery rates of Plasmodium falciparum parasitaemia from longitudinal data. Bulletin of World Health Organization 1976; 54: 685-693.

13. Sama W, Dietz K, Smith T. Distribution of survival times of deliberate Plasmodium falciparum infections in tertiary syphilis patients. Transactions of the Royal Society Tropical Medicine and Hygiene 2006; 100: 811-816.

14. Singer B, Cohen JE. Estimating malaria incidence and recovery rates from panel surveys. Mathematical Biosciences 1980; 49: 273-305.

15. Smith TA. Estimation of heterogeneity in malaria transmission by stochastic modelling of apparent deviations from mass action kinetics. Malaria Journal 2008; 7: 12.

16. Smith DL, et al. Ross, Macdonald, and a theory for the dynamics and control of mosquito-transmitted pathogens. PLoS Pathogens 2012; 8 (4). doi: 10.1371/journal.ppat.1002588.

17. White MT, et al. Heterogeneity in malaria exposure and vaccine response: implications for the interpretation of vaccine efficacy trials. Malaria Journal 2010; 9: 82. doi: 10.1186/1475-2875-9-82.

18. Ross R. Report on the Prevention of Malaria in Mauritius. New York: E. P. Dutton \& Company, 1908.

19. Keeling MJ, Rohan P. Modeling Infectious Diseases in Humans and Animals. Princeton, New Jersey: Princeton University Press, 2008.

20. Aguas R, et al. Prospects for malaria eradication in Sub-Saharan Africa. PLoS ONE 2008; 3 (3): e1767. doi: 10.1371/journal.pone.0001767.

21. Hens N, et al. Modeling Infectious Disease Parameters based on Serological and Social Contact Data: A Modern Statistical Perspective. New York: Springer, 2012.

22. Ross R. Application of the theory of probabilities to the study of priori pathometry. In: Proceedings of the Royal Society of London. Series A, Containing Papers of a Mathematical and Physical Character, 1916.

23. de Smith MJ. Statistical Analysis Handbook - A Web-based Statistics Resource. Winchelsea, UK: Winchelsea Press, 2015.

24. Molenberghs G, Verbeke G. Models for Discrete Longitudinal Data. New York: Springer, Series in Statistics, 2005.

25. Zhang DW, Lin XH. Variance component testing in generalized linear mixed models for longitudinal/ clustered data and other related topics. Random Effect and Latent Variable Model Selection 2008; 192: 19-36.

26. Faes C, et al. Estimating herd-specific force of infection by using random-effects models for clustered binary data and monotone fractional polynomials. Journal of the Royal Statistical Society Series C:Applied Statistics 2006; 55: 595-613.

27. Shkedy Z, et al. Modelling age-dependent force of infection from prevalence data using fractional polynomials. Statistics in Medicine 2006; 25: 1577-1591.

28. Akaike H. New look at statistical-model identification. IEEE Transactions on Automatic Control 1974; Ac19: 716-723.

29. Musca SC, et al. Data with hierarchical structure: impact of intraclass correlation and sample size on type-I error. Frontiers in Psychology 2011; 2: 74.

30. Wu S, Crespi CM, Wong WK. Comparison of methods for estimating the intraclass correlation coefficient for binary responses in cancer prevention cluster randomized trials. Contemporary Clinical Trials 2012; 33: 869-880.

31. Bretscher MT, et al. The distribution of Plasmodium falciparum infection durations. Epidemics 2011; 3: 109-118.

32. Schwarz GE. Estimating the dimension of a model. Annals of Statistics 1978; 6(2): 461-464.

33. Verbeke G, Molenberghs G. Linear Mixed Models for Longitudinal Data. New York: Springer, 2000.

34. Wang J, Xie H, Fisher JH. Multilevel Models. Applications Using SAS. Berlin: Higher Education Press and Walter de Gruyter GmbH \& Co. KG, 2012.

35. Riley EM, et al. Do maternally acquired antibodies protect infants from malaria infection? Parasite Immunology 2001; 23: 51-59.

36. Walldorf JA, et al. School-age children are a reservoir of Malaria Infection in Malawi. PLoS ONE 2015; 10: e0134061.

37. Poschl B, et al. Comparative diagnosis of malaria infections by microscopy, nested PCR, and LAMP in northern Thailand. American Journal of Tropical Medicine and Hygiene 2010; 83: 56-60.

38. Coleman RE, et al. Comparison of PCR and microscopy for the detection of asymptomatic malaria in a Plasmodium falciparum/vivax endemic area in Thailand. Malaria Journal 2006; 5: 121. doi: 10.1186/1475-2875-5-121.

39. Tan KS, French B, Troxel AB. Regression modeling of longitudinal data with outcome-dependent observation times: extensions and comparative evaluation. Statistics in Medicine 2014; 33: 4770-4789.

40. Rizopoulos D, Verbeke G, Molenberghs G. Shared parameter models under random effects misspecification. Biometrika 2008; 95: 63-74.

\section{APPENDIX}

Though, fractional polynomials are very flexible, they can result into negative estimates for the FOI whenever the estimated probability to be infected before age $a$ is a non-monotone function [21, 27]. A solution 
Table A1. Overview of the fractional polynomial model selection

\begin{tabular}{|c|c|c|c|c|c|c|c|c|c|}
\hline Power & -3 & -2 & -1 & -0.5 & 0 & 0.5 & 1 & 2 & 3 \\
\hline AIC & $7202 \cdot 3$ & $7178 \cdot 6$ & $7150 \cdot 0$ & $7152 \cdot 9$ & $7154 \cdot 4$ & $7160 \cdot 9$ & $7171 \cdot 2$ & $7190 \cdot 6$ & $7204 \cdot 9$ \\
\hline
\end{tabular}

Table A2. Overview of model building (number of observations in each case equal to 8645)

\begin{tabular}{|c|c|c|c|}
\hline Model & Log-likelihood & AIC & $\mathrm{BIC}$ \\
\hline$a^{-1} \times S+l \times S+S+P T+P T \times S+b_{1 i j}+b_{2 j} \times S$ & $-3199 \cdot 09$ & $6442 \cdot 17$ & $6525 \cdot 75$ \\
\hline$a^{-1} \times S+l \times S+S+P T+P T \times S+b_{2 j} \times S$ & $-3208 \cdot 24$ & $6458 \cdot 48$ & $6538 \cdot 26$ \\
\hline$a^{-1} \times S+l \times S+S+P T+P T \times S+b_{1 i j}+b_{2 j}$ & $-3213 \cdot 90$ & $6467 \cdot 80$ & $6543 \cdot 78$ \\
\hline $\boldsymbol{a}^{-1} \times \boldsymbol{S}+\boldsymbol{l} \times \boldsymbol{S}+\boldsymbol{S}+\boldsymbol{P} \boldsymbol{T}+b_{1 i \boldsymbol{j}}+b_{2 \boldsymbol{j}} \times S$ & $-3204 \cdot 93$ & $6441 \cdot 86$ & $6502 \cdot 64$ \\
\hline$a^{-1}+l \times S+S+P T+b_{1 i j}+b_{2 j} \times S$ & $-3210 \cdot 56$ & $6449 \cdot 12$ & $6502 \cdot 31$ \\
\hline$a^{-1} \times S+l+S+P T+b_{1 i j}+b_{2 j} \times S$ & $-3209 \cdot 73$ & $6447 \cdot 45$ & $6500 \cdot 64$ \\
\hline$a^{-1}+l+S+P T+b_{1 i j}+b_{2 j} \times S$ & $-3211 \cdot 87$ & $6447 \cdot 74$ & $6493 \cdot 32$ \\
\hline
\end{tabular}

$S$, study site; $P$, infection status at previous visit; $T$, treatment with AL at previous infection; $P T$, combination of $P$ and $T$. Note that $P$ and $T$ were collinear (sign of $T$ changes whenever $P$ is included with $T$ ).
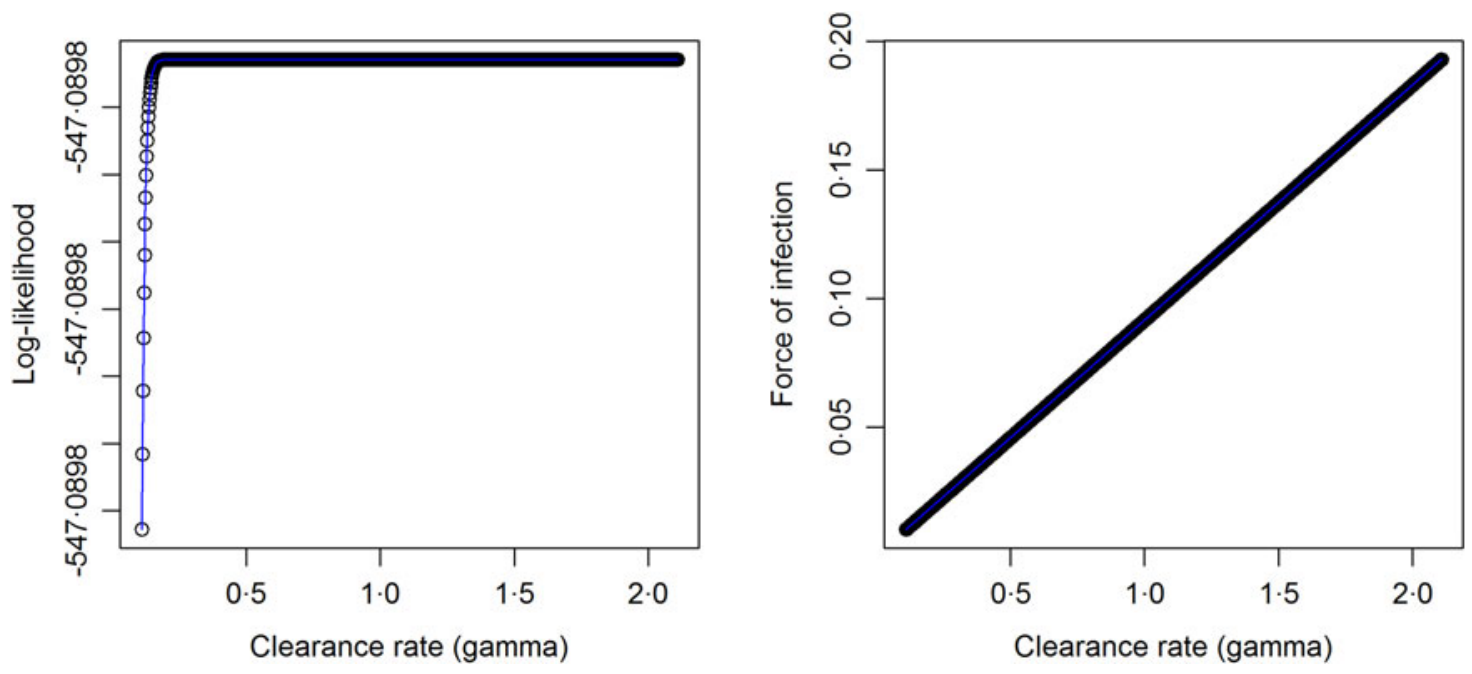

Fig. A1. Plots for log-likelihood versus the clearance rate (left panel) and FOI versus the clearance rate (right panel) obtained after fitting 1000 models to the data according to $\pi=\lambda /(\lambda+\gamma)\left(1-e^{-(\lambda+\gamma) a}\right)$ as given by Pull and Grab (1974) by choosing values for the annual clearance rate on a grid of $0 \cdot 1$ to $2 \cdot 0$ with a step size of $0 \cdot 0019$.

to this is to define a non-negative FOI, $\lambda_{l}\left(a_{i j k} \mid b_{i}\right) \geqslant 0$ for all $a$ and to estimate $\pi_{l}\left(a_{i j k} \mid b_{i}\right)$ under these constraints [27]. From Table 1, for a logit link function, the condition $\eta^{\prime}\left(a_{i j k} \mid b_{i}\right) \geqslant-\gamma /\left(1-\pi_{l}\left(a_{i j k} \mid b_{i}\right)\right)$ should be satisfied as to estimate a positive FOI. One option is to fit a constrained FP to ensure the above condition holds by applying a constraint on parameter estimates depending on the functional relationship with age. However, this approach becomes challenging especially if it involves constraining random effects. An alternative option is to find a probability of estimating a negative FOI using the model results. If this probability is considerably small, say less than $0 \cdot 01$, then one can consider the first option unnecessary. In this paper, the second option was applied. Indeed, all sitespecific coefficients for age effect were negative (see Table 3), meaning that the site-specific derivatives for the linear predictors, $\eta^{\prime}\left(a_{i j k} \mid b_{i}\right)=\left(-\left(\hat{\beta}_{6}\right) a_{i j k}^{-2}\right.$ $\left.-\left(\hat{\beta}_{7}\right) a_{i j k}^{-2},-\left(\hat{\beta}_{8}\right) a_{i j k}^{-2}\right)>0$. This implies that the above condition always holds in our case since $a_{i j k}^{-2}$, 

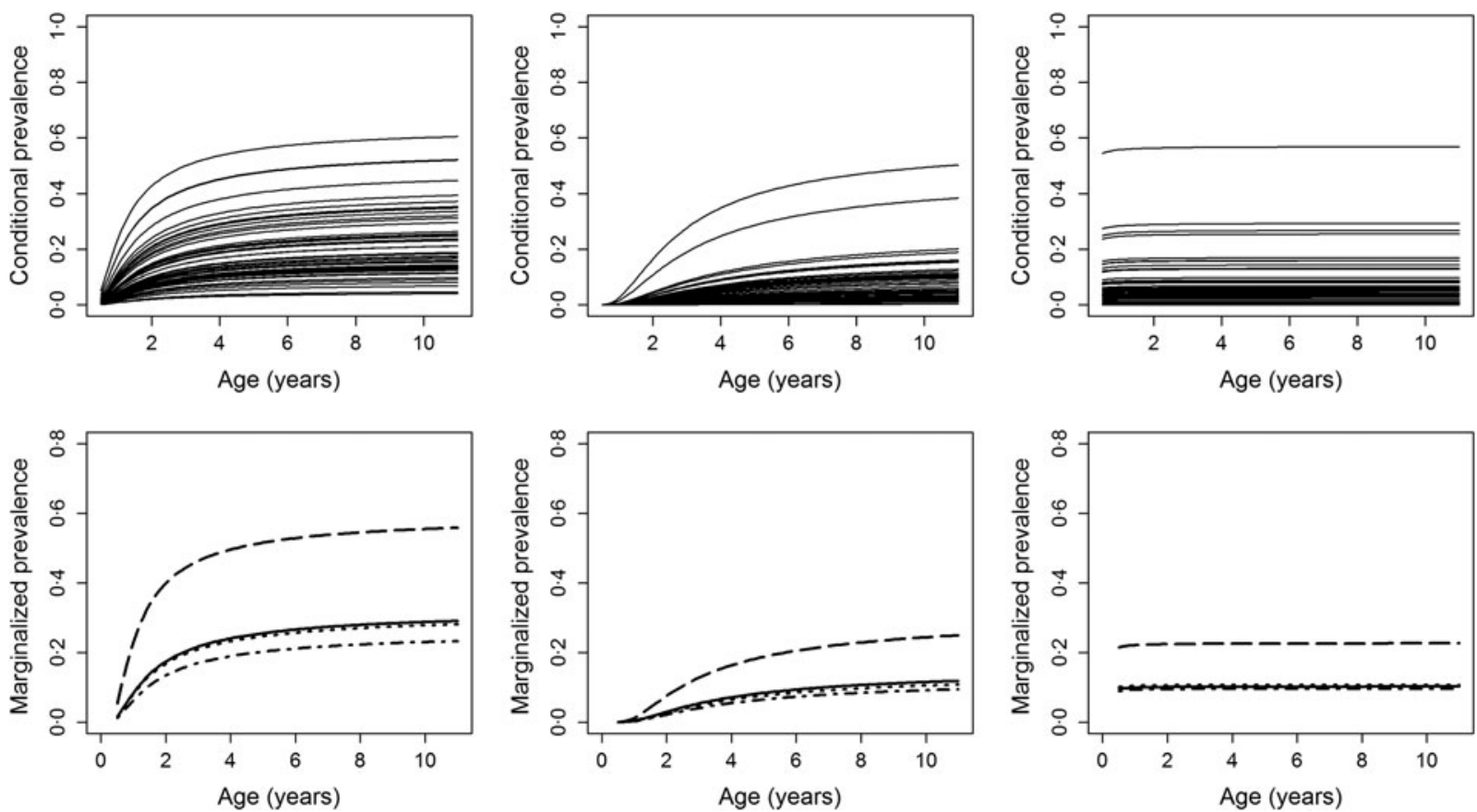

Fig. A2. Top row: Individual-specific evolutions for the conditional prevalence, by study site for children assumed to be symptomatic at the previous visit and were born in the baseline year (2001). Bottom row: Average evolutions for marginalized prevalence, by study site and the infection status at the previous visit and past use of AL (negative and no AL in the past (solid lines), negative and AL in the past (dotted lines), symptomatic (dash-dotted lines) and asymptomatic (long-dashed lines)). Left panel: Nagongera, middle panel: Kihihi, right panel: Walukuba.

Table A3; Maximum values for the marginal annual FOI by study site, previous infection status and use of AL, and by age group

\begin{tabular}{|c|c|c|c|c|}
\hline \multirow[b]{2}{*}{ Site } & \multirow[b]{2}{*}{ Previous infection status and use of $\mathrm{AL}$} & \multicolumn{3}{|c|}{ Maximum annual FOI } \\
\hline & & $<1$ year & $1-4$ years & $5-10$ years \\
\hline \multirow[t]{4}{*}{ Nagongera } & Negative, no AL & 3.99 & $4 \cdot 21$ & $8 \cdot 49$ \\
\hline & Negative, AL & $4 \cdot 45$ & $4 \cdot 80$ & $9 \cdot 69$ \\
\hline & Symptomatic & $2 \cdot 21$ & $2 \cdot 07$ & $4 \cdot 14$ \\
\hline & Asymptomatic & $7 \cdot 73$ & $9 \cdot 21$ & $18 \cdot 70$ \\
\hline \multirow[t]{4}{*}{ Kihihi } & Negative, no AL & $5 \cdot 35$ & $24 \cdot 95$ & $64 \cdot 82$ \\
\hline & Negative, $\mathrm{AL}$ & $1 \cdot 46$ & $4 \cdot 64$ & 11.78 \\
\hline & Symptomatic & 1.06 & $3 \cdot 23$ & $8 \cdot 11$ \\
\hline & Asymptomatic & $4 \cdot 62$ & $20 \cdot 25$ & $52 \cdot 56$ \\
\hline \multirow[t]{4}{*}{ Walukuba } & Negative, no AL & $18 \cdot 01$ & $6 \cdot 65$ & $11 \cdot 28$ \\
\hline & Negative, AL & $20 \cdot 07$ & $7 \cdot 41$ & $12 \cdot 58$ \\
\hline & Symptomatic & $8 \cdot 02$ & $2 \cdot 95$ & $5 \cdot 01$ \\
\hline & Asymptomatic & $98 \cdot 24$ & $36 \cdot 34$ & $61 \cdot 66$ \\
\hline
\end{tabular}

$\gamma$ and $\left(1-\pi_{l}\left(a_{i j k} \mid b_{i}\right)\right)$ are always positive. Therefore, the probability to estimate a negative FOI was zero.

For example, based on model results in Table 3, the conditional age-time dependent FOI for a subject from Walukuba, born in the baseline year (2001, that is, shifted year of birth $=0$ ) and was symptomatic at the previous visit can be estimated as follows,

$$
\begin{aligned}
\hat{\lambda}_{0}\left(a_{i j k} \mid b_{i}\right)= & \hat{\gamma} \operatorname{Exp}\left(\hat{\beta}_{0}+\hat{\beta}_{6} a_{i j k}^{-1}+\hat{\beta}_{4}+\mathrm{b}_{1 i j}+\mathrm{b}_{21 j}\right) \\
& -\left(\hat{\beta}_{6}\right) a_{i j k}^{-2 *} \hat{\pi}_{0}\left(\mathrm{a}_{i j k} \mid b_{i}\right)
\end{aligned}
$$

where $\hat{\beta}_{0}=-3.04, \hat{\beta}_{6}=-0.05, \quad \hat{\beta}_{4}=-0.24$, and 
Table A4; Marginal FOI and the 95\% confidence bounds for the age- and time-dependent marginal annual FOI by study site, previous infection status and use of AL, and by age group for children born in the baseline year (2001)

\begin{tabular}{|c|c|c|c|c|}
\hline $\begin{array}{l}\text { Infection status at the previous } \\
\text { visit and past use of AL }\end{array}$ & $\begin{array}{l}\text { Age in } \\
\text { years }\end{array}$ & $\begin{array}{l}\text { Nagongera } \\
\text { Marginal annual FOI } \\
(95 \% \text { CI }) \times 1000\end{array}$ & $\begin{array}{l}\text { Kihihi } \\
\text { Marginal annual FOI } \\
(95 \% \text { CI }) \times 1000\end{array}$ & $\begin{array}{l}\text { Walukuba } \\
\text { Marginal annual FOI } \\
(95 \% \text { CI }) \times 1000\end{array}$ \\
\hline \multicolumn{5}{|l|}{ Lower bound } \\
\hline \multirow[t]{3}{*}{ Negative and no AL in the past } & $<1$ & $143 \cdot 78(141 \cdot 16-146 \cdot 39)$ & $9 \cdot 27(8 \cdot 52-10 \cdot 01)$ & $10 \cdot 20(9 \cdot 75-10 \cdot 65)$ \\
\hline & $1-4$ & $53 \cdot 69(53 \cdot 20-54 \cdot 19)$ & $22 \cdot 69(22 \cdot 34-23 \cdot 04)$ & $0.95(0.92-0.97)$ \\
\hline & $5-10$ & $8.57(8 \cdot 53-8 \cdot 62)$ & $7 \cdot 24(7 \cdot 17-7 \cdot 31)$ & $0.09(0 \cdot 09-0.09)$ \\
\hline \multirow[t]{3}{*}{ Negative and AL in the past } & $<1$ & $137 \cdot 35(134 \cdot 84-139 \cdot 87)$ & $7 \cdot 64(7 \cdot 28-8 \cdot 00)$ & $10 \cdot 72(10 \cdot 27-11 \cdot 18)$ \\
\hline & $1-4$ & $51 \cdot 67(51 \cdot 19-52 \cdot 14)$ & $20 \cdot 09(19 \cdot 82-20 \cdot 35)$ & $0.99(0.97-1 \cdot 02)$ \\
\hline & $5-10$ & $8 \cdot 29(8 \cdot 24-8 \cdot 33)$ & $6 \cdot 59(6 \cdot 52-6 \cdot 65)$ & $0 \cdot 10(0 \cdot 09-0 \cdot 10)$ \\
\hline \multirow[t]{3}{*}{ Symptomatic } & $<1$ & $105 \cdot 62(103 \cdot 73-107 \cdot 51)$ & $6 \cdot 26(5 \cdot 98-6 \cdot 54)$ & $9 \cdot 58(9 \cdot 18-9 \cdot 98)$ \\
\hline & $1-4$ & $41 \cdot 4(41 \cdot 02-41 \cdot 79)$ & $16 \cdot 91(16 \cdot 70-17 \cdot 12)$ & $0 \cdot 89(0 \cdot 87-0 \cdot 91)$ \\
\hline & $5-10$ & $6 \cdot 83(6 \cdot 79-6 \cdot 87)$ & $5 \cdot 70(5 \cdot 65-5 \cdot 75)$ & $0.09(0.08-0 \cdot 09)$ \\
\hline \multirow[t]{3}{*}{ Asymptomatic } & $<1$ & $426 \cdot 73(420 \cdot 32-433 \cdot 14)$ & $24 \cdot 87(23 \cdot 68-26 \cdot 06)$ & $22 \cdot 88(22 \cdot 14-23 \cdot 63)$ \\
\hline & $1-4$ & $123 \cdot 3(122 \cdot 22-124 \cdot 39)$ & $55 \cdot 20(54 \cdot 57-55 \cdot 81)$ & $2 \cdot 11(2 \cdot 07-2 \cdot 14)$ \\
\hline & $5-10$ & $16 \cdot 86(16 \cdot 78-16 \cdot 93)$ & $15 \cdot 69(15 \cdot 57-55 \cdot 83)$ & $0 \cdot 20(0 \cdot 20-0 \cdot 20)$ \\
\hline
\end{tabular}

\begin{tabular}{lllll} 
Upper bound & \multicolumn{5}{l}{} \\
\hline Negative and no AL in the past & $<1$ & $234 \cdot 51(229 \cdot 74-239 \cdot 28)$ & $12 \cdot 22(11 \cdot 16-13 \cdot 28)$ & $309 \cdot 33(285 \cdot 17-333 \cdot 49)$ \\
& $1-4$ & $224 \cdot 99(223 \cdot 32-226 \cdot 65)$ & $61 \cdot 66(60 \cdot 13-63 \cdot 20)$ & $112 \cdot 84(109 \cdot 37-116 \cdot 31)$ \\
& $5-10$ & $445 \cdot 73(442 \cdot 83-448 \cdot 62)$ & $161 \cdot 20(157 \cdot 32-165 \cdot 08)$ & $191 \cdot 40(186 \cdot 57-196 \cdot 22)$ \\
Negative and AL in the past & $<1$ & $223 \cdot 74(219 \cdot 15-216 \cdot 36)$ & $10 \cdot 03(9 \cdot 54-10 \cdot 53)$ & $322 \cdot 88(298 \cdot 09-347 \cdot 66)$ \\
& $1-4$ & $214 \cdot 75(213 \cdot 15-216 \cdot 36)$ & $51 \cdot 65(50 \cdot 91-52 \cdot 39)$ & $117 \cdot 76(114 \cdot 2-121 \cdot 31)$ \\
Symptomatic & $5-10$ & $424 \cdot 49(421 \cdot 70-427 \cdot 29)$ & $131 \cdot 29(129 \cdot 73-132 \cdot 85)$ & $199 \cdot 73(194 \cdot 78-204 \cdot 67)$ \\
& $<1$ & $170 \cdot 65(167 \cdot 29-174 \cdot 0)$ & $8 \cdot 22(7 \cdot 84-8 \cdot 60)$ & $246 \cdot 55(231 \cdot 99-261 \cdot 10)$ \\
Asymptomatic & $1-4$ & $164 \cdot 17(163 \cdot 02-165 \cdot 32)$ & $42 \cdot 74(42 \cdot 17-43 \cdot 30)$ & $89 \cdot 53(87 \cdot 46-91 \cdot 61)$ \\
& $5-10$ & $320 \cdot 14(318 \cdot 21-322 \cdot 07)$ & $107 \cdot 71(106 \cdot 53-108 \cdot 89)$ & $151 \cdot 64(148 \cdot 75-154 \cdot 52)$ \\
& $<1$ & $741 \cdot 36(728 \cdot 10-754 \cdot 61)$ & $32 \cdot 81(31 \cdot 15-34 \cdot 46)$ & $1134 \cdot 5(1034 \cdot 6-1234 \cdot 4)$ \\
& $1-4$ & $717 \cdot 36(712 \cdot 29-722 \cdot 31)$ & $159 \cdot 84(157 \cdot 55-162 \cdot 14)$ & $417 \cdot 93(403 \cdot 49-432 \cdot 36)$ \\
& $5-10$ & $1532 \cdot 75(1523 \cdot 4-1542 \cdot 1)$ & $429 \cdot 11(423 \cdot 66-434 \cdot 55)$ & $711 \cdot 13(691 \cdot 0-731 \cdot 26)$ \\
\hline \hline
\end{tabular}

$\hat{\pi}_{0}\left(a_{i j k} \mid b_{i}\right)$ is the corresponding age-time conditional prevalence given as,

$\hat{\pi}_{0}\left(a_{i j k} \mid b_{i}\right)=\frac{\operatorname{Exp}\left(\hat{\beta}_{0}+\hat{\beta}_{6} a_{i j k}^{-1}+\hat{\beta}_{4}+b_{1 i j}+b_{21 j}\right)}{1+\operatorname{Exp}\left(\hat{\beta}_{0}+\hat{\beta}_{6} a_{i j k}^{-1}+\hat{\beta}_{4}+b_{1 i j}+b_{21 j}\right)}$

and $\hat{\gamma}$ is an estimate for the clearance rate. The conditional FOI for other sites given the infection status at the previous visit and past use of AL can be estimated in a similar way.

\section{MARGINALISATION}

A sample of $M=1000$ of the random affects vector $b_{i}=\left(b_{1}, b_{2 s j}\right)^{T}, s=1,2,3$ (sites), was generated from a multi-variate normal distribution, $\mathrm{N}\left(0, \hat{\mathrm{L}} \hat{\mathrm{L}}^{T}\right)$, where for example, for Walukuba, $\hat{\mathrm{L}}=(0.49,1.67)^{T}$ whose elements are the square roots of $\hat{d}_{11}$ and $\hat{d}_{22}$, respectively as given in Table 3. A fine grid of age, $a=0.5$ to 11 with interval $0 \cdot 1$ years (the age range in the data, though extrapolation is possible) was considered. For example, the marginalized FOI at each age value in the grid, again considering a subject from Walukuba, born in the baseline year and was symptomatic at the previous visit is calculated as in (9).

$$
\begin{aligned}
\hat{\lambda}_{0}(a)= & \frac{1}{1000} \sum_{i=1}^{1000}\left(\hat { \gamma } \operatorname { E x p } \left(\hat{\beta}_{0}+\hat{\beta}_{6} a^{-1}+\hat{\beta}_{4}+b_{1 i}\right.\right. \\
& \left.\left.+b_{21 i}\right)\right)-\left(\hat{\beta}_{6}\right) a^{-2 *} \hat{\pi}_{0}(a)
\end{aligned}
$$

where $\hat{\pi}_{0}(a)$ is the corresponding marginalized prevalence given by

$$
\begin{aligned}
\hat{\pi}_{0}(a) & =\frac{1}{1000} \\
& \sum_{i=1}^{1000}\left(\frac{\operatorname{Exp}\left(\hat{\beta}_{0}+\hat{\beta}_{6} a^{-1}+\hat{\beta}_{4}+b_{1 i}+b_{21 i}\right)}{1+\operatorname{Exp}\left(\hat{\beta}_{0}+\hat{\beta}_{6} a^{-1}+\hat{\beta}_{4}+b_{1 i}+b_{21 i}\right)}\right)
\end{aligned}
$$


Extensions to estimate the marginal averages at different birth years, for different study sites and for different infection statuses at the previous visit, are straightforward. The SAS macro performing the numerical averaging for a case of $\hat{\gamma}=1.643$ is attached in the Appendix.

\section{A GENERAL S(I)J(R)S SYSTEM}

Let $s, i$ and $r$ represent the proportion susceptible, infected and recovered, respectively. Also, let $\mu$ represent the natural birth rate assumed to be equal to the natural death rate, $\beta$ the transmission rate, $\gamma$ the clearance rate and $\sigma$ the recovery rate.

System:

$$
\begin{gathered}
\frac{\mathrm{d} s}{\mathrm{~d} t}=\mu-\beta s i+\sigma r-\mu s \\
\frac{\mathrm{d} i_{1}}{\mathrm{~d} t}=\beta s i-\gamma i_{1}-\mu i_{1}, \\
\frac{\mathrm{d} i_{2}}{\mathrm{~d} t}=\gamma i_{1}-\gamma i_{2}-\mu i_{2}, \\
\cdot \\
\cdot \\
\frac{\mathrm{d} i_{J}}{\mathrm{~d} t}=\gamma i_{J-1}-\gamma i_{J}-\mu i_{J}, \\
\frac{\mathrm{d} r}{\mathrm{~d} t}=\gamma i_{J}-\sigma r-\mu r
\end{gathered}
$$

where $i=\sum_{j=1}^{J} i_{j}$
Rewriting the system collapsing the infectious classes into $i$ :

$$
\begin{aligned}
& \frac{\mathrm{d} s}{\mathrm{~d} t}=\mu-\beta s i+\sigma r-\mu s, \\
& \frac{\mathrm{d} i}{\mathrm{~d} t}=\beta s i-\gamma i_{J}-\mu i, \\
& \frac{\mathrm{d} r}{\mathrm{~d} t}=\gamma i_{J}-\sigma r-\mu r,
\end{aligned}
$$

Simplifying the model to an $\mathbf{S}(\mathbf{I})_{\mathbf{J}} \mathbf{S}$ system:

$$
\begin{aligned}
& \frac{\mathrm{d} s}{\mathrm{~d} t}=\mu-\beta s i+\gamma i_{J}-\mu s, \\
& \frac{\mathrm{d} i}{\mathrm{~d} t}=\beta s i-\gamma i_{J}-\mu i,
\end{aligned}
$$

yields (replacing dildt by $i^{\prime}, \lambda=\beta i$ and $s=1-i$ )

$i^{\prime}=\lambda(1-i)-\gamma i_{J}-\mu i$

and thus

$\lambda=\frac{i^{\prime}+\gamma i_{J}+\mu i}{1-i} \approx \frac{i^{\prime}+\gamma i_{J}}{1-i}$,

expressing time dependency,

$\lambda(t)=\frac{i^{\prime}(t)+\gamma i_{J}(t)+\mu i(t)}{1-i(t)} \approx \frac{i^{\prime}(t)+\gamma i_{J}(t)}{1-i(t)}$,

since $\mu i(t) \ll \gamma i_{J}(t)$. Let's look at the factor $\gamma i_{J}(t)$. In case $J=1, \gamma i_{J}(t)=\gamma i(t)$. In case $J>1, \gamma i_{J}(t)<\gamma i(t)$. This gives us a lower and upper boundary for our FOI.

$\left[\lambda_{L}(t), \lambda_{U}(t)\right]=\left[\frac{i^{\prime}(t)}{1-i(t)}, \frac{i^{\prime}(t)+\gamma i(t)}{1-i(t)}\right]$

These formulas readily extend to the ageheterogeneous case since we do not explicitly model the underlying transmission mechanism.

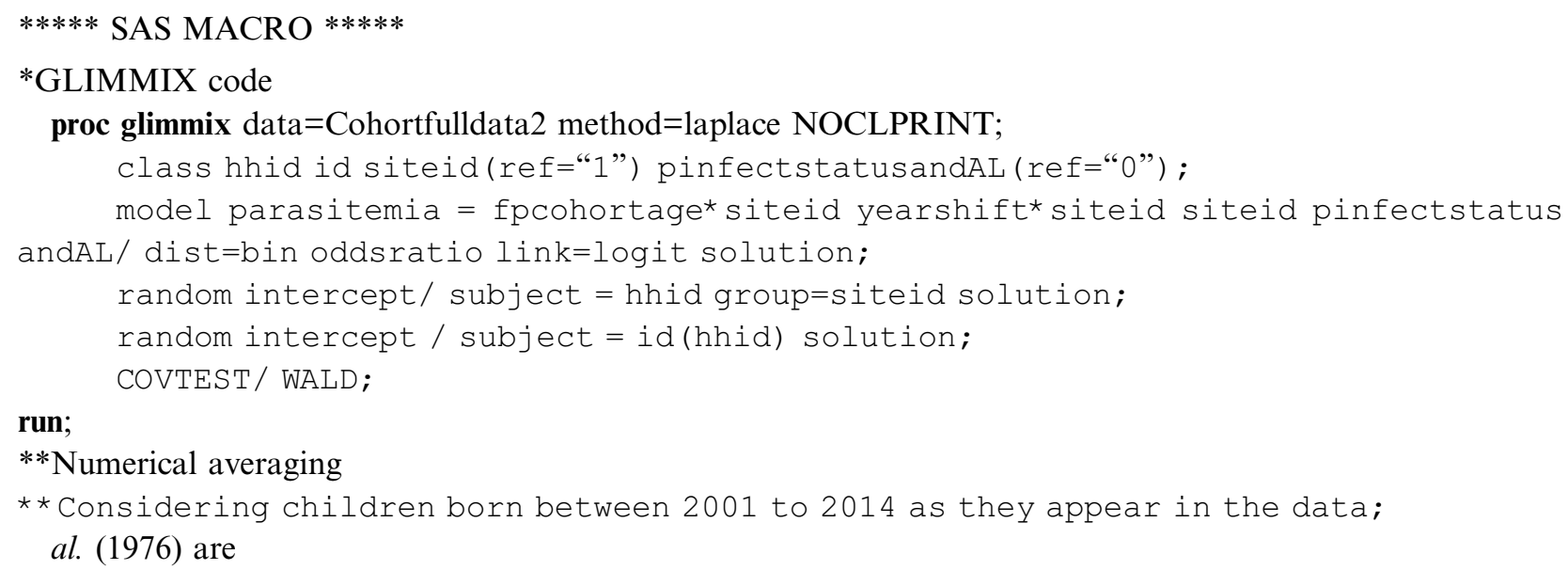

data numaveragingprevfoinc; 
do site $=\mathbf{1}$ to $\mathbf{3}$ by $\mathbf{1}$; *study sites 1(walukuba),2(kihihi),3(nagongera);

do pinfect $=\mathbf{1}$ to $\mathbf{4}$ by $\mathbf{1}$; *infection status 1 (negative+no AL), 2(negative+AL), 3(symptomatic), 4 (asymptomatic);

do subject $\mathbf{1}$ to $\mathbf{1 0 0 0}$ by $\mathbf{1}$; *generate 1000 samples;

bi1=rannor (123); bi2=rannor(123); bi3=rannor(123); bi4=rannor(123); *used seed=123 to generate from standard normal;

$\mathrm{d} 11=\mathbf{0 . 2 4} ; \mathrm{d} 22=\mathbf{2 . 8 0} ; \mathrm{d} 33=\mathbf{1 . 1 6} ; \mathrm{d} 44=\mathbf{0 . 2 1} ; *$ variances from the final fit, elements in $\mathrm{D}$;

$\mathrm{rd} 11=\mathrm{d} 11 * * \mathbf{0 . 5} ; \mathrm{rd} 22=\mathrm{d} 22 * * \mathbf{0 . 5} ; \mathrm{rd} 33=\mathrm{d} 33 * * \mathbf{0 . 5} ; \mathrm{rd} 44=\mathrm{d} 44 * * \mathbf{0 . 5} ; * \operatorname{sqrt}(\mathrm{S} 2)$ to be used in Cholesky decomposition;

$r 1=r d 11 * b i 1 ; \quad r 2=r d 22 * b i 2 ; r 3=r d 33 * b i 3 ; r 4=r d 44 * b i 4 ; * u s i n g$ U+sqrt (s2)*rannor

(seed): Note elements in here are sqrt of elements in D;

do a $=\mathbf{0 . 5}$ to $\mathbf{1 1}$ by $\mathbf{0 . 1}$; *generate 1000 samples at each age point in the grid;

do $\mathrm{L}=\mathbf{0}$ to $\mathbf{1 3}$ by $\mathbf{1}$; *Repeat the above process for each value of birth year shift ( $\mathrm{L}=$ year of birth -2001 );

* Parameter estimates;

$\mathrm{B} 0=-\mathbf{3 . 0 4} ; \mathrm{B} 1=0.86 ; \mathrm{B} 2=\mathbf{2 . 1 9} ; \mathrm{B} 3=-0.01 ; \mathrm{B} 4=-0.24 ; \mathrm{B} 5=1.23 ; \mathrm{B} 6=-0.05 ; \mathrm{B} 7=-4.01 ; \mathrm{B} 8=-1.75 ; \mathrm{B} 9=-0.13$;

$\mathrm{B} 10=\mathbf{0 . 1 1} ; \mathrm{B} 11=\mathbf{0 . 0 4} ; \quad \mathrm{ap}=\mathbf{1} / \mathrm{a} ;{ }^{*}$ Power of age, age-1;

* Linear Predictors;

$1 \mathrm{p} 11=\mathrm{B} 0+\mathrm{B} 6 * \mathrm{ap}+\mathrm{B} 9 * \mathrm{~L}+\mathrm{r} 1+\mathrm{r} 2 ; \quad \mathrm{p} 12=\mathrm{B} 0+\mathrm{B} 6 * \mathrm{ap}+\mathrm{B} 9 * \mathrm{~L}+\mathrm{B} 3+\mathrm{r} 1+\mathrm{r} 2$;

$1 \mathrm{p} 13=\mathrm{B} 0+\mathrm{B} 6 * \mathrm{ap}+\mathrm{B} 9 * \mathrm{~L}+\mathrm{B} 4+\mathrm{r} 1+\mathrm{r} 2 ; \mathrm{lp} 14=\mathrm{B} 0+\mathrm{B} 6 * \mathrm{ap}+\mathrm{B} 9 * \mathrm{~L}+\mathrm{B} 5+\mathrm{r} 1+\mathrm{r} 2$;

$1 \mathrm{p} 21=\mathrm{B} 0+\mathrm{B} 7 * \mathrm{ap}+\mathrm{B} 10 * \mathrm{~L}+\mathrm{B} 1+\mathrm{r} 1+\mathrm{r} 3 ; \mathrm{lp} 22=\mathrm{B} 0+\mathrm{B} 7 * \mathrm{ap}+\mathrm{B} 10 * \mathrm{~L}+\mathrm{B} 1+\mathrm{B} 3+\mathrm{r} 1+\mathrm{r} 3$;

$1 \mathrm{p} 23=\mathrm{B} 0+\mathrm{B} 7 * \mathrm{ap}+\mathrm{B} 10 * \mathrm{~L}+\mathrm{B} 1+\mathrm{B} 4+\mathrm{r} 1+\mathrm{r} 3 ; \mathrm{lp} 24=\mathrm{B} 0+\mathrm{B} 7 * \mathrm{ap}+\mathrm{B} 10 * \mathrm{~L}+\mathrm{B} 1+\mathrm{B} 5+\mathrm{r} 1+\mathrm{r} 3$;

$1 \mathrm{p} 31=\mathrm{B} 0+\mathrm{B} 8 * \mathrm{ap}+\mathrm{B} 11^{*} \mathrm{~L}+\mathrm{B} 2+\mathrm{r} 1+\mathrm{r} 4 ; \mathrm{lp} 32=\mathrm{B} 0+\mathrm{B} 8 * \mathrm{ap}+\mathrm{B} 11^{*} \mathrm{~L}+\mathrm{B} 2+\mathrm{B} 3+\mathrm{r} 1+\mathrm{r} 4$;

lp33=B0+B8* ap+B11* L+B2+B4+r1+r4; lp34=B0+B8*ap+B11* L+B2+B5+r1+r4;

* Derivative of linear predictor;

lpder $1=-(B 6)$ * $(a p * a p) ; 1 p d e r 2=-(B 7)$ * $(a p * a p) ; 1 p d e r 3=-(B 8)$ * (ap*ap) ;

*Prevalence;

if site $=\mathbf{1}$ and pinfect $=\mathbf{1}$ then $\mathrm{p}=\exp (\operatorname{lp} 11) /(\mathbf{1}+\exp (\operatorname{lp} 11))$;

if site $=\mathbf{1}$ and pinfect $=\mathbf{2}$ then $\mathrm{p}=\exp (\operatorname{lp} 12) /(\mathbf{1}+\exp (\operatorname{lp} 12))$;

if site $=\mathbf{1}$ and pinfect $=\mathbf{3}$ then $\mathrm{p}=\exp (\operatorname{lp} 13) /(\mathbf{1}+\exp (\operatorname{lp} 13))$;

if site $=\mathbf{1}$ and pinfect $=\mathbf{4}$ then $\mathrm{pi}=\exp (\operatorname{lp} 14) /(\mathbf{1}+\exp (\operatorname{lp} 14))$;

if site $=\mathbf{2}$ and pinfect $=\mathbf{1}$ then $\mathrm{pi}=\exp (\operatorname{lp} 21) /(\mathbf{1}+\exp (\operatorname{lp} 21))$;

if site $=\mathbf{2}$ and pinfect $=\mathbf{2}$ then $\mathrm{p} i=\exp (\operatorname{lp} 22) /(\mathbf{1}+\exp (\operatorname{lp} 22))$;

if site $=\mathbf{2}$ and pinfect $=\mathbf{3}$ then $\mathrm{pi}=\exp (\operatorname{lp} 23) /(\mathbf{1}+\exp (\operatorname{lp} 23))$;

if site $=\mathbf{2}$ and pinfect $=\mathbf{4}$ then $\mathrm{pi}=\exp (\operatorname{lp} 24) /(\mathbf{1}+\exp (\operatorname{lp} 24))$;

if site $=\mathbf{3}$ and pinfect $=\mathbf{1}$ then $\mathrm{p}=\exp (\operatorname{lp} 31) /(\mathbf{1}+\exp (\operatorname{lp} 31))$;

if site $=\mathbf{3}$ and pinfect $=\mathbf{2}$ then $\mathrm{pi}=\exp (\operatorname{lp} 32) /(\mathbf{1}+\exp (\operatorname{lp} 32))$;

if site $=\mathbf{3}$ and pinfect $=\mathbf{3}$ then $\mathrm{p} i=\exp (\operatorname{lp} 33) /(\mathbf{1}+\exp (\operatorname{lp} 33))$;

if site $=\mathbf{3}$ and pinfect $=\mathbf{4}$ then $\mathrm{p}=\exp (\operatorname{lp} 34) /(\mathbf{1}+\exp (\operatorname{lp} 34))$;

$\star * \mathrm{FOI}$;

* Clearance rate of 1.643 for children <1 year as given by Bekessy et al. (1976) is demon-

strated, a similar code can easily be adopted for ages $1-4$ years and $5-10$ years.;

if site $=\mathbf{1}$ and pinfect $=\mathbf{1}$ and $\mathrm{a}<\mathbf{1}$ then foi $=\mathbf{1 . 6 4 3} * \exp (\operatorname{lp} 11)+\operatorname{lpder} 1 * \exp (\operatorname{lp} 11) /(\mathbf{1}+\exp (\operatorname{lp} 11))$;

if site $=\mathbf{1}$ and pinfect $=\mathbf{2}$ and $\mathrm{a}<\mathbf{1}$ then foi $=\mathbf{1 . 6 4 3} * \exp (1 \mathrm{p} 12)+\operatorname{lpder} 1 * \exp (\operatorname{lp} 12) /(\mathbf{1}+\exp (\operatorname{lp} 12))$;

if site $=\mathbf{1}$ and pinfect $=\mathbf{3}$ and $\mathrm{a}<\mathbf{1}$ then foi $=\mathbf{1 . 6 4 3} * \exp (\operatorname{lp} 13)+1 \mathrm{pder} 1 * \exp (\operatorname{lp} 13) /(\mathbf{1}+\exp (\operatorname{lp} 13))$;

if site $=\mathbf{1}$ and pinfect $=\mathbf{4}$ and $\mathrm{a}<\mathbf{1}$ then foi $=\mathbf{1 . 6 4 3} * \exp (\operatorname{lp} 14)+\operatorname{lpder} 1 * \exp (\operatorname{lp} 14) /(\mathbf{1}+\exp (\operatorname{lp} 14))$;

if site $=\mathbf{2}$ and pinfect $=\mathbf{1}$ and $\mathrm{a}<\mathbf{1}$ then foi $=\mathbf{1 . 6 4 3} * \exp (\operatorname{lp} 21)+\operatorname{lpder} 2 * \exp (\operatorname{lp} 21) /(\mathbf{1}+\exp (\operatorname{lp} 21))$;

if site $=\mathbf{2}$ and pinfect $=\mathbf{2}$ and $\mathrm{a}<\mathbf{1}$ then foi $=\mathbf{1 . 6 4 3} * \exp (\operatorname{lp} 22)+1 \mathrm{pder} 2 * \exp (\operatorname{lp} 22) /(\mathbf{1}+\exp (\operatorname{lp} 22))$;

if site $=\mathbf{2}$ and pinfect $=\mathbf{3}$ and $\mathrm{a}<\mathbf{1}$ then foi $=\mathbf{1 . 6 4 3} * \exp (\operatorname{lp} 23)+\operatorname{lpder} 2 * \exp (\operatorname{lp} 23) /(\mathbf{1}+\exp (\operatorname{lp} 23))$;

if site $=\mathbf{2}$ and pinfect $=\mathbf{4}$ and $\mathrm{a}<\mathbf{1}$ then foi $=\mathbf{1 . 6 4 3} * \exp (\operatorname{lp} 24)+1 \mathrm{pder} 2 * \exp (\operatorname{lp} 24) /(\mathbf{1}+\exp (\operatorname{lp} 24))$;

if site $=\mathbf{3}$ and pinfect $=\mathbf{1}$ and $a<\mathbf{1}$ then foi $=\mathbf{1 . 6 4 3} * \exp (\operatorname{lp} 31)+1$ pder $3 * \exp (\operatorname{lp} 31) /(\mathbf{1}+\exp (\operatorname{lp} 31))$;

if site $=\mathbf{3}$ and pinfect $=\mathbf{2}$ and $\mathrm{a}<\mathbf{1}$ then foi $=\mathbf{1 . 6 4 3} * \exp (\operatorname{lp} 32)+\operatorname{lpder} 3 * \exp (\operatorname{lp} 32) /(\mathbf{1}+\exp (\operatorname{lp} 32))$; 
if site $=\mathbf{3}$ and pinfect $=\mathbf{3}$ and $\mathrm{a}<\mathbf{1}$ then foi $=\mathbf{1 . 6 4 3} * \exp (1 \mathrm{p} 33)+1 \mathrm{pder} 3 * \exp (\operatorname{lp} 33) /(\mathbf{1}+\exp (\operatorname{lp} 33))$;

if site $=\mathbf{3}$ and pinfect $=\mathbf{4}$ and $\mathrm{a}<\mathbf{1}$ then foi $=\mathbf{1 . 6 4 3} * \exp (1 \mathrm{p} 34)+1 \mathrm{pder} 3 * \exp (\operatorname{lp} 34) /(\mathbf{1}+\exp (1 \mathrm{p} 34))$;

output;

end;

end;

end;

end;

end;

run;

* sort data;

proc sort data = numaveragingprevfoinc; by a site pinfect L;run;

* Get means ;

proc means data $=$ numaveragingprevfoinc; var pi foi; by a site pinfect L; output out=outpifoinc; run; * Keep data for marginalized means;

data marginalizedprevandfoinc; set outpifoinc; where _stat_='MEAN'; run; 